\title{
Effective and energy-preserving time discretization for a general nonlinear poromechanical formulation
}

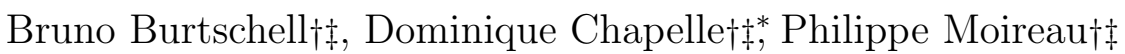 \\ $\dagger$ Inria, Université Paris-Saclay \\ $\ddagger$ Laboratoire de Mécanique des Solides, Ecole Polytechnique, CNRS, Université Paris-Saclay
}

Computers and Structures 182 (2017) 313-324

\begin{abstract}
We consider a general nonlinear poromechanical model, formulated based on fundamental thermodynamics principle, suitable for representing the coupling of rapid internal fluid flows with large deformations of the solid, and compatible with a wide class of constitutive behavior. The objective of the present work is to propose for this model a time discretization scheme of the partitioned type, to allow the use of existing time schemes - and possibly separate solvers - for each component of the model, i.e. for the fluid and the solid. To that purpose, we adapt and extend an earlier proposed approach devised for fluid-structure interaction in an Arbitrary Lagrangian-Eulerian framework. We then establish an energy estimate for the resulting time scheme, in a form that is consistent with the underlying energy principle in the poromechanical formulation, up to some numerical dissipation effects and some perturbations that we have carefully identified and assessed. In addition, we provide some numerical illustrations of our numerical strategy with test problems that present typical features of large strains and rapid fluid flows, and also a case of singular transition related to total drainage. An example of challenging application envisioned for this model and associated numerical coupling scheme concerns the perfusion of the heart.
\end{abstract}

\section{Introduction}

Recently, novel challenging applications such as cardiac modeling have required the introduction of general formulations coupling porous flows and hyperelastic formulations, and compatible with large displacements, finite strains and strong inertial effects both in the solid and in the fluid. In this context, a general poromechanics formulation was proposed in [10] based on fundamental thermodynamics principles, see also [41] where the same type of model was subsequently considered. As inertia effects and large displacements are considered, the final formulation is very similar to the coupling of hyperelastic dynamics for the skeleton - i.e., the solid constituent - with a conservative form of the so-called Arbitrary Lagrangian-Eulerian (ALE) formulation of the compressible Navier-Stokes equations [15, 33] set on the same domain, hence, with a domain velocity given by the skeleton physical velocity. The compressible analogy comes from the product of the fluid volume fraction with the fluid density that, together, play the role of a varying fluid density. Finally, when compared with standard fluid-structure interaction (FSI) problems, we have the additional distributed coupling term representing the interaction between the two phases $[6,13,14]$. From this analogy, [10] introduced a time scheme with an energy balance at the time-discrete level inspired from the work [32] initially devoted to

\footnotetext{
${ }^{*}$ Corresponding author: dominique.chapelle@inria.fr
} 
classical fluid-structure interaction problems. The proposed time scheme combined in a monolithic formulation a mid-point energy-conserving extension of the mid-point Newmark scheme and a second-order Crank-Nicolson scheme for the fluid- with an additional specific treatment of the Darcy term discretization in order to respect the energy balance in the fluid-skeleton interaction. This scheme was proved in [10] to be second-order accurate and unconditionally stable, similarly to its initial fluid-structure counterpart in [32].

However, this scheme has some drawbacks when considering its practical use in simulation software - in industrial codes in particular - as it implies the use of a Newton-Raphson solution procedure on a monolithic fluid+solid formulation. Therefore, we propose in the present article an alternative time discretization inspired from state-of-the-art partitioned FSI time-schemes $[18,19,27]$. Partitioned solvers aim at solving the interaction problem by coupling independent solvers for the fluid and the solid $[2,3,21,28,29,35]$. Therefore, they are much more modular than monolithic approaches and allow the use of existing legacy software [30]. However, the computational efficiency of partitioned approaches compared with a monolithic approach must be assessed [4, 8, 27, 28]. Hence, the question of monolithic versus partitioned approaches has already been raised in other specific poromechanics formulations, typically with Darcy flows [34].

As we aim at relying on a classical Newmark scheme for the solid with an energy-conserving extension for general hyperelastic laws $[22,25]$, we set out in this article to propose our timescheme based on the recent partitioned FSI scheme of [1]. This scheme combines a Newmark scheme for the solid [22] with an effective Chorin-Temam projection scheme in the fluid [11, 23, 39]. The fluid viscous sub-step, taking into account the convective-viscous effects and the geometrical non-linearities, is treated explicitly. Moreover, at each time step the projection sub-step is implicitly coupled with the structure with Robin coupling conditions derived from Nitsche's interface method $[7,36]$. The specificity of this coupling strategy is twofold. First, it allows to prove stability independently of the added-mass effect typically present in blood flow simulations, which in particular is known to compromise the stability of explicit coupling - time-marching - schemes, see [8]. Note that this added-mass effect has also been evidenced in poroelastic models with an impact that directly correlates with fluid fraction [5]. Secondly, the coupling strategy of [1] is, to our best knowledge, the only time scheme that allows for non-linear conservative time-stepping within a 3D general solid, as opposed to the more direct Dirichlet-Neumann semi-implicit coupling [19]. From this starting point, we propose in the present article a partitioned scheme adapted to the poromechanics formulation of [10], with an adequate treatment of the additional fluid fraction variable, and a specific treatment of the distributed coupling conditions. The resulting scheme is proved to satisfy a discrete energy estimate, hence, to be unconditionally stable. Compared with [1] from which we draw the inspiration of our time scheme, our major contributions lie in

- extending this time scheme to our more complex case of a two-phase poromechanical problem;

- establishing the discrete energy estimate with the total free energy of the mixture, in a general nonlinear framework.

Furthermore, as our proposed method has the same algorithmic complexity as that of [1], we can similarly expect very significant gains in computational efficiency compared to a monolithic approach, as already assessed numerically in [19], in particular.

The paper is organized as follows. In Section 2, we recall the formulation of the general poromechanical model of [10] that we consider, with the associated energy balance. Next, in Section 3 we introduce our proposed partitioned time discretization scheme, and we provide a 
detailed stability analysis of this time scheme by establishing a discrete energy estimate. One ingredient of this analysis is an adapted form of the so-called "geometric conservation law" $[16,17,38,40]$, which in our case is shown to be satisfied by construction, up to perturbations induced by spatial discretization that we analyse in details. In Section 4 , we provide some implementation considerations, and several numerical illustrations for representative test problems proposed in the recent literature [9]. In addition, we present a test case in which we precisely monitor the energy balance and quantitatively assess the various sources of perturbations induced by spatial discretization. Finally, we give some concluding remarks in Section 5 .

\section{Poromechanical formulation}

\subsection{Basic definitions}

We consider the general poromechanical model proposed in [10]. This is a two-phase mixture type model, in which a fluid phase and a solid phase are assumed to coexist and interact at each point, $\phi$ denoting the volume fraction of the fluid phase - also called the porosity.

The solid phase is primarily described by the displacement field $\underline{y}_{\mathrm{s}}(\underline{\xi}, t)$ defined at every point $\xi$ in the (fixed) reference domain $\Omega^{0}$, and at any time $t$ in the time window considered. We will use the corresponding velocity field

$$
\underline{v}_{\mathrm{s}}=\frac{\mathrm{d} \underline{y}_{\mathrm{s}}}{\mathrm{dt}}=\partial_{t} \underline{y}_{\mathrm{s}}(\underline{\xi}, t) .
$$

The displacement field maps the reference domain $\Omega^{0}$ to the deformed domain $\Omega_{t}$, viz.

$$
\underline{\xi} \in \Omega^{0} \mapsto \underline{\mathrm{x}}=\underline{\xi}+\underline{y}_{\mathrm{S}}(\underline{\xi}, t),
$$

and the associated deformation gradient tensor is

$$
\underline{\underline{F}}=\underline{\underline{\mathbb{1}}}+\underline{\underline{\nabla}} \underline{\underline{\xi}} \underline{y_{\mathrm{s}}},
$$

with determinant $J=\operatorname{det} \underline{\underline{F}}$. We point out that $J$ represents the local change of volume of the global mixture, whereas the change of volume of the solid phase itself is given by $J(1-$ $\phi) /\left(1-\phi_{0}\right)$, with $\phi_{0}$ the fluid volume fraction in the undeformed configuration, and we define $J_{\mathrm{s}}=J(1-\phi)$. We recall the definitions of the right Cauchy-Green deformation tensor and of the Green-Lagrange strain tensor, i.e., respectively,

$$
\underline{\underline{C}}=\underline{\underline{F}}^{T} \cdot \underline{\underline{F}}, \quad \underline{\underline{e}}=\frac{1}{2}(\underline{\underline{C}}-\underline{\underline{\mathbb{1}}}) .
$$

The mass per unit volume of the solid phase in the reference configuration is denoted by $\rho_{\mathrm{s} 0}$.

The internal fluid flow is represented by the velocity $\underline{v}_{\mathrm{f}}$ and pressure $p$, both fields being naturally defined in the deformed domain $\Omega_{t}$. The fluid is assumed to be incompressible, hence, the fluid mass per unit volume $\rho_{\mathrm{f}}$ is constant. The quantity $m$ is defined as the added fluid mass per unit volume of the reference configuration, i.e.

$$
m=\rho_{\mathrm{f}}\left(J \phi-\phi_{0}\right) .
$$

The fluid is assumed to be Newtonian, with the usual decomposition of the fluid Cauchy stress tensor into viscous and hydrostatic contributions, i.e.

$$
\underline{\underline{\sigma}}_{\mathrm{f}}=\underline{\underline{\sigma}}_{\mathrm{vis}}\left(\underline{v}_{\mathrm{f}}\right)-p \underline{\underline{\mathbb{1}}} .
$$


Recalling the classical transformation rule from the Cauchy stress tensor to the second Piola-Kirchhoff stress tensor

$$
\underline{\underline{\underline{\Sigma}}}=J \underline{\underline{F}}^{-1} \cdot \underline{\underline{\sigma}} \cdot \underline{\underline{F}}^{-T}
$$

here written for the global stress tensors of the mixture, we will denote by $\underline{\underline{\Sigma}}_{s}$ the contribution of the solid in the second Piola-Kirchhoff stress tensor $\underline{\underline{\Sigma}}$, i.e.

$$
\underline{\underline{\Sigma}}_{s}=\underline{\underline{\Sigma}}-\phi J \underline{\underline{F}}^{-1} \cdot \underline{\underline{\sigma}}_{\mathrm{f}} \cdot \underline{\underline{F}}^{-T}=\underline{\underline{\Sigma}}-\phi \underline{\underline{\Sigma}}_{\mathrm{vis}}+\phi p J \underline{\underline{C}}^{-1},
$$

with $\underline{\underline{\underline{\Sigma}}}_{\mathrm{vis}}=J \underline{\underline{F}}^{-1} \cdot \underline{\underline{\sigma}}_{\mathrm{vis}} \cdot \underline{\underline{F}}^{-T}$, see Section 2.4 below for more detailed specifications of the constitutive laws.

\subsection{Strong formulation}

The strong form of the poromechanical model reads [10]

$$
\left\{\begin{array}{cc}
\rho_{\mathrm{s} 0}\left(1-\phi_{0}\right) \frac{\mathrm{d} \underline{v}_{\mathrm{s}}}{\mathrm{dt}}-\underline{\nabla}_{\underline{\xi}} \cdot\left(\underline{\underline{F}} \cdot \underline{\underline{\Sigma}}_{\mathrm{s}}\right)+p J \underline{\underline{F}}^{-T} \cdot \underline{\nabla}_{\underline{\xi}} \phi & \\
-J \phi^{2} \underline{\underline{k}}_{\mathrm{f}}^{-1} \cdot\left(\underline{v}_{\mathrm{f}}-\underline{v}_{\mathrm{s}}\right)=\rho_{\mathrm{s} 0}\left(1-\phi \underline{\phi}_{0}\right) \underline{f}, & \text { in } \Omega^{0}, \\
\frac{1}{J} \frac{\mathrm{d}}{\mathrm{dt}}\left(\rho_{\mathrm{f}} J \phi \underline{v}_{\mathrm{f}}\right)+\underline{\nabla}_{\underline{\mathrm{x}}} \cdot\left(\rho_{\mathrm{f}} \phi \underline{v}_{\mathrm{f}} \otimes \rho_{\mathrm{f}}\left(\underline{v}_{\mathrm{f}}-\underline{v}_{\mathrm{s}}\right)\right)-\theta \underline{v}_{\mathrm{f}} & \\
+\phi^{2} \underline{k}_{\mathrm{f}}^{-1} \cdot\left(\underline{v}_{\mathrm{f}}-\underline{v}_{\mathrm{s}}\right)-\underline{\nabla}_{\underline{\mathrm{x}}} \cdot\left(\phi \underline{\sigma}_{\mathrm{vis}}\right)+\phi \underline{\nabla}_{\underline{\mathrm{x}}} p=\rho_{\mathrm{f}} \phi \underline{f}, & \text { in } \Omega_{t}, \\
\frac{1}{J} \frac{\mathrm{d}}{\mathrm{dt}}\left(J \rho_{\mathrm{f}} \phi\right)+\underline{\nabla}_{\underline{\mathrm{x}}} \cdot\left(\rho_{\mathrm{f}} \phi\left(\underline{v}_{\mathrm{f}}-\underline{v}_{\mathrm{s}}\right)\right)=\theta, & \text { in } \Omega_{t}
\end{array}\right.
$$

where $\underline{\underline{k}}_{\mathrm{f}}$ denotes the so-called permeability tensor that governs the friction forces between the solid and fluid phases (we will also use its inverse $\underline{\underline{D}}_{\mathrm{f}}$ ), $f$ the applied distributed force per unit mass, and $\theta$ the fluid mass input per unit volume in the deformed configuration that may be used in some problems to model some specific inflow $(\theta>0)$ or outflow $(\theta<0)$ conditions. This is a coupled system, albeit in essence the first equation governs the solid deformation, the second the fluid flow, and the third the fluid mass conservation. Note in passing the slight abuse of notation - that we will repeatedly use throughout the paper - by which we employ the same notation for fields defined over the domains $\Omega^{0}$ and $\Omega_{t}-$ e.g., for the velocity fields - which means that composition by the deformation mapping or its inverse is implicitly used.

\section{Remark 1 (Comparison with fluid-structure interaction)}

From a formal standpoint the above system bears some interesting resemblance with a fluidstructure interaction problem written in the Arbitrary Lagrangian-Eulerian (ALE) formalism, see e.g. [1]. The main differences here are that the fluid and the solid interact everywhere - and not only on boundaries - via the distributed friction term $J \phi^{2} \underline{\underline{k}}_{\mathrm{f}}^{-1} \cdot\left(\underline{v}_{\mathrm{f}}-\underline{v}_{\mathrm{S}}\right)$ and the porosity gradient term $p J \underline{F}^{-T} \cdot \underline{\nabla}_{\xi} \phi$, the ALE domain velocity is substituted with the physical solid velocity, and the fluid mass conservation is made more complex due to the combination of fluid and solid at every point (with fluid volume fraction $\phi$ ). Nevertheless, the similarities will allow us to draw some inspiration from a previously-proposed time scheme to design our discrete problem, i.e. [1].

Of course, the above equations must be complemented with adequate boundary conditions. Denoting by $\underline{t}$ the total traction on the boundary of the domain $\Omega_{t}$, and by $\underline{t}_{0}=J\left\|\underline{\underline{F}}^{-T} \cdot \underline{n}_{0}\right\| \underline{t}$ the transported counterpart on the boundary of the reference domain $\Omega^{0}$, as in [10] we will consider: 
- Dirichlet boundary conditions for both phases, i.e. prescribed skeleton displacements and fluid velocities

$$
\underline{y}_{\mathrm{s}}=\underline{y}_{\mathrm{s}}^{\mathrm{pr}}, \quad \underline{v}_{\mathrm{f}}=\underline{v}_{\mathrm{f}}^{\mathrm{pr}},
$$

on the subpart of the boundary that we denote by $\Gamma_{D}^{0}$ in the reference configuration and $\Gamma_{D}^{t}$ in the current configuration;

- Neumann boundary conditions - namely, prescribed forces - for both phases together, with proportional repartition ${ }^{1}$ of boundary traction

$$
\underline{\underline{\sigma}} \cdot \underline{\underline{n}}=\underline{t} \Leftrightarrow \underline{\underline{F}} \cdot \underline{\underline{\Sigma}} \cdot \underline{n}_{0}=\underline{t}_{0}, \quad \underline{\sigma}_{\mathrm{f}} \cdot \underline{n}=\underline{t},
$$

on $\Gamma_{N}^{0}\left(\right.$ or $\left.\Gamma_{N}^{t}\right)$;

- Neumann boundary condition for the global mixture, but vanishing fluid flux and proportional repartition of tangential boundary traction

$$
\underline{\underline{\sigma}} \cdot \underline{n}=\underline{t} \Leftrightarrow \underline{\underline{F}} \cdot \underline{\underline{\Sigma}} \cdot \underline{n}_{0}=\underline{t}_{0}, \quad \pi_{\tau}\left(\underline{\underline{\sigma}}_{\mathrm{f}} \cdot \underline{n}\right)=\pi_{\tau}(\underline{t}), \quad\left(\underline{v}_{\mathrm{f}}-\underline{v}_{\mathrm{s}}\right) \cdot \underline{n}=0
$$

on $\Gamma_{N \text { nof }}^{0}\left(\right.$ or $\Gamma_{N \text { nof }}^{t}$ ), where $\pi_{\tau}=\underline{\underline{\mathbb{1}}}-\underline{n} \otimes \underline{n}$ denotes the projection onto the tangential plane;

- Neumann boundary condition for the global mixture with fluid velocity coinciding with the solid velocity (no sliding)

$$
\underline{\underline{\sigma}} \cdot \underline{n}=\underline{t} \Leftrightarrow \underline{\underline{F}} \cdot \underline{\underline{\Sigma}} \cdot \underline{n}_{0}=\underline{t}_{0}, \quad \underline{v}_{\mathrm{f}}=\underline{v}_{\mathrm{s}},
$$

on $\Gamma_{N \text { nos }}^{0}\left(\right.$ or $\left.\Gamma_{N \text { nos }}^{t}\right)$.

\subsection{Weak formulation}

We consider test functions $\left(\underline{v}_{\mathrm{s}}^{*}, \underline{v}_{\mathrm{f}}^{*}, q^{*}\right)$ associated with the main unknowns $\left(\underline{y}_{\mathrm{s}}, \underline{v}_{\mathrm{f}}, m\right)$ and satisfying similar Dirichlet boundary conditions, albeit in a homogeneous form, i.e.

$$
\left.\underline{v}_{\mathrm{s}}^{*}\right|_{\Gamma_{D}^{0}}=\left.\underline{v}_{\mathrm{f}}^{*}\right|_{\Gamma_{D}^{0}}=0,\left.\quad\left(\left(\underline{v}_{\mathrm{f}}^{*}-\underline{v}_{\mathrm{s}}^{*}\right) \cdot \underline{n}\right)\right|_{\Gamma_{N \mathrm{nof}}^{0}}=0,\left.\quad\left(\underline{v}_{\mathrm{f}}^{*}-\underline{v}_{\mathrm{s}}^{*}\right)\right|_{\Gamma_{N \mathrm{nos}}^{0}}=0 .
$$

Multiplying System (2) by these test functions and integrating space-wise yields [10]

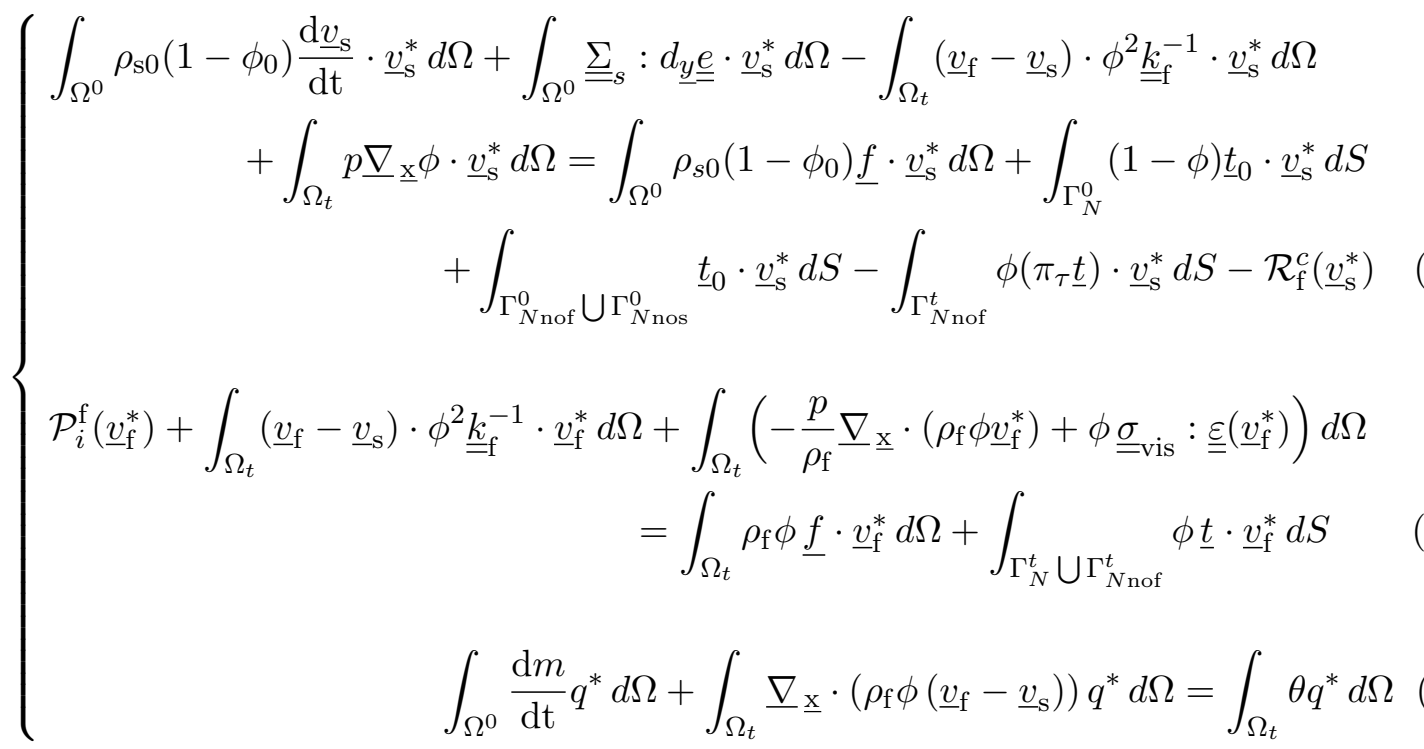

\footnotetext{
${ }^{1}$ proportionality is meant here according to the respective volume fractions of the two phases
} 
with

$$
\mathcal{P}_{i}^{\mathrm{f}}\left(\underline{v}^{*}\right)=\int_{\Omega^{0}} \frac{\mathrm{d}}{\mathrm{dt}}\left(J \rho_{\mathrm{f}} \phi \underline{v}_{\mathrm{f}}\right) \cdot \underline{v}^{*} d \Omega+\int_{\Omega_{t}} \underline{\nabla}_{\underline{\mathrm{x}}} \cdot\left(\rho_{\mathrm{f}} \phi \underline{v}_{\mathrm{f}} \otimes\left(\underline{v}_{\mathrm{f}}-\underline{v}_{\mathrm{s}}\right)\right) \cdot \underline{v}^{*} d \Omega-\int_{\Omega_{t}} \theta \underline{v}_{\mathrm{f}} \cdot \underline{v}^{*} d \Omega,
$$

and the residual representing the fluid reaction forces

$$
\begin{aligned}
\mathcal{R}_{\mathrm{f}}^{c}\left(\underline{v}_{\mathrm{s}}^{*}\right)=\mathcal{P}_{i}^{\mathrm{f}}\left(\underline{v}_{\mathrm{s}}^{*}\right)+\int_{\Omega_{t}}\left(\underline{v}_{\mathrm{f}}-\underline{v}_{\mathrm{s}}\right) & \cdot \phi^{2} \underline{\underline{k}}_{\mathrm{f}}^{-1} \cdot \underline{v}_{\mathrm{s}}^{*} d \Omega+\int_{\Omega_{t}}\left(-\frac{p}{\rho_{\mathrm{f}}} \underline{\nabla}_{\mathrm{x}} \cdot\left(\rho_{\mathrm{f}} \phi \underline{v}_{\mathrm{s}}^{*}\right)+\phi \underline{\sigma}_{\mathrm{vis}}: \underline{\underline{\varepsilon}}\left(\underline{v}_{\mathrm{s}}^{*}\right)\right) d \Omega \\
& -\int_{\Omega_{t}} \rho_{\mathrm{f}} \phi \underline{f} \cdot \underline{v}_{\mathrm{s}}^{*} d \Omega-\int_{\Gamma_{N}^{t}} \phi \underline{t} \cdot \underline{v}_{\mathrm{s}}^{*} d S-\int_{\Gamma_{N \text { nof }}^{t}} \phi\left(\pi_{\tau} \underline{t}\right) \cdot \underline{v}_{\mathrm{s}}^{*} d S .
\end{aligned}
$$

This weak formulation characterizes the main unknowns $\left(\underline{y}_{\mathrm{s}}, \underline{v}_{\mathrm{f}}, m\right)$, from which all other quantities can be computed. In particular, we have $\phi=\frac{m / \rho_{\mathrm{f}}+\phi_{0}}{J\left(\underline{y}_{\mathrm{s}}\right)}$, and the pressure $p$ will be given by a constitutive equation, see next section. Note that - as is usual in computational mechanics, especially in a nonlinear framework - we do not dwell on the mathematical definition of the functional spaces, typically considered to be Sobolev spaces of the form $W^{1, s}$ with $s$ sufficiently large for all integrals in (3) to be well-defined, see e.g. [12, 31] and references therein for more details.

In order to transform (3a) into a more compact form, we define the following pseudo-residual based on the above-introduced residual

$$
\begin{aligned}
\overline{\mathcal{R}}_{\mathrm{f}}^{c}\left(\underline{v}_{\mathrm{s}}^{*}\right) & =\mathcal{R}_{\mathrm{f}}^{c}\left(\underline{v}_{\mathrm{s}}^{*}\right)-\int_{\Omega_{t}}\left(\underline{v}_{\mathrm{f}}-\underline{v}_{\mathrm{s}}\right) \cdot \phi^{2} \underline{\underline{k}}_{\mathrm{f}}^{-1} \cdot \underline{v}_{\mathrm{s}}^{*} d \Omega+\int_{\Omega_{t}} p \underline{\nabla}_{\underline{\mathrm{x}}} \phi \cdot \underline{v}_{\mathrm{s}}^{*} d \Omega \\
& =\mathcal{P}_{i}^{\mathrm{f}}\left(\underline{v}_{\mathrm{s}}^{*}\right)+\int_{\Omega_{t}}\left(-p \phi \underline{\nabla} \underline{\mathrm{x}}_{\underline{\mathrm{x}}} \cdot \underline{v}_{\mathrm{s}}^{*}+\phi \underline{\sigma}_{\mathrm{vis}}: \underline{\underline{\varepsilon}}\left(\underline{v}_{\mathrm{s}}^{*}\right)\right) d \Omega-\int_{\Omega_{t}} \rho_{\mathrm{f}} \phi \underline{f} \cdot \underline{v}_{\mathrm{s}}^{*} d \Omega \\
& -\int_{\Gamma_{N}^{t}} \phi \underline{t} \cdot \underline{v}_{\mathrm{s}}^{*} d S-\int_{\Gamma_{N \text { nof }}^{t}} \phi\left(\pi_{\tau} \underline{t}\right) \cdot \underline{v}_{\mathrm{s}}^{*} d S,
\end{aligned}
$$

where we have used the identity $\underline{\nabla}_{\underline{\mathrm{x}}} \cdot\left(\phi \underline{v}_{\mathrm{S}}^{*}\right)=\phi \underline{\nabla}_{\underline{\mathrm{x}}} \cdot \underline{v}_{\mathrm{S}}^{*}+\underline{\nabla}_{\underline{\mathrm{x}}} \phi \cdot \underline{v}_{\mathrm{s}}^{*}$. We infer the following alternative form for $(3 \mathrm{a})$

$$
\begin{aligned}
& \int_{\Omega^{0}} \rho_{\mathrm{s} 0}\left(1-\phi_{0}\right) \frac{\mathrm{d} \underline{v}_{\mathrm{s}}}{\mathrm{dt}} \cdot \underline{v}_{\mathrm{s}}^{*} d \Omega+\int_{\Omega^{0}} \underline{\underline{\Sigma}}_{s}: d_{\underline{y}} \underline{\underline{e}} \cdot \underline{v}_{\mathrm{s}}^{*} d \Omega=\int_{\Omega^{0}} \rho_{s 0}\left(1-\phi_{0}\right) \underline{f} \cdot \underline{v}_{\mathrm{s}}^{*} d \Omega \\
& +\int_{\Gamma_{N}^{0}}(1-\phi) \underline{t}_{0} \cdot \underline{v}_{\mathrm{s}}^{*} d S+\int_{\Gamma_{N \text { nof }}^{0} \cup \Gamma_{N \text { nos }}^{0}} \underline{t}_{0} \cdot \underline{v}_{\mathrm{s}}^{*} d S-\int_{\Gamma_{N \text { nof }}^{t}} \phi\left(\pi_{\tau} \underline{t}\right) \cdot \underline{v}_{\mathrm{s}}^{*} d S-\overline{\mathcal{R}}_{\mathrm{f}}^{c}\left(\underline{v}_{\mathrm{s}}^{*}\right) .
\end{aligned}
$$

\subsection{Constitutive laws and energy balance}

We consider a total stress tensor given by [10]

$$
\underline{\underline{\Sigma}}=\phi \underline{\underline{\underline{\Sigma}}} \text { vis }+\left.\frac{\partial \Psi(\underline{\underline{e}}, m)}{\partial \underline{\underline{e}}}\right|_{m}+\left.\frac{\partial \Psi^{\mathrm{damp}}(\underline{\underline{e}}, \underline{\underline{\underline{e}}})}{\partial \underline{\underline{\underline{e}}}}\right|_{\underline{\underline{e}}}
$$

with $\Psi(\underline{\underline{e}}, m)$ the Helmholtz free energy of the mixture, and $\Psi^{\text {damp }}(\underline{\underline{e}}, \underline{\underline{e}})$ a viscous pseudopotential. Due to fluid incompressibility, $\Psi(\underline{\underline{e}}, m)=\Psi_{\mathrm{s}}\left(\underline{\underline{e}}, J_{\mathrm{s}}\right)$ with $\Psi_{\mathrm{s}}$ the solid free energy - meaning that the fluid cannot store any energy - hence, we have

$$
\underline{\underline{\Sigma}}=\phi \underline{\underline{\underline{\Sigma}}}_{\mathrm{vis}}+\left.\frac{\partial \Psi_{\mathrm{s}}\left(\underline{\underline{e}}, J_{\mathrm{s}}\right)}{\partial \underline{\underline{e}}}\right|_{J_{\mathrm{s}}}+\left.\frac{\partial \Psi^{\mathrm{damp}}(\underline{\underline{e}}, \underline{\underline{\dot{e}}})}{\partial \underline{\underline{e}}}\right|_{\underline{\underline{e}}}-p J \underline{\underline{C}}^{-1}
$$


see [10]. Recalling (1), this gives for the solid contribution

$$
\begin{aligned}
\underline{\underline{\Sigma}}_{\mathrm{s}} & =\left.\frac{\partial \Psi(\underline{\underline{e}}, m)}{\partial \underline{\underline{e}}}\right|_{m}+\left.\frac{\partial \Psi^{\mathrm{damp}}(\underline{\underline{e}}, \underline{\underline{e}})}{\partial \underline{\underline{e}}}\right|_{\underline{\underline{e}}}+\phi p J \underline{\underline{C}}^{-1} \\
& =\left.\frac{\left.\partial \Psi_{\mathrm{s}} \underline{\underline{\underline{e}}}, J_{\mathrm{s}}\right)}{\partial \underline{\underline{e}}}\right|_{J_{\mathrm{s}}}+\left.\frac{\partial \Psi^{\mathrm{damp}}(\underline{\underline{e}}, \underline{\underline{\underline{e}}})}{\partial \underline{\underline{\underline{e}}}}\right|_{\underline{\underline{e}}}-(1-\phi) p J \underline{\underline{C}}^{-1} .
\end{aligned}
$$

In addition, we have

$$
p=\left.\rho_{\mathrm{f}} \frac{\partial \Psi(\underline{\underline{e}}, m)}{\partial m}\right|_{\underline{\underline{e}}}=-\left.\frac{\partial \Psi_{\mathrm{s}}\left(\underline{\underline{e}}, J_{\mathrm{s}}\right)}{\partial J_{\mathrm{s}}}\right|_{\underline{\underline{e}}} .
$$

Defining the total kinetic energy of the system

$$
\mathcal{K}=\frac{1}{2} \int_{\Omega^{0}} \rho_{\mathrm{s} 0}\left(1-\phi_{0}\right) \underline{v}_{\mathrm{s}}^{2} d \Omega+\frac{1}{2} \int_{\Omega_{t}} \rho_{\mathrm{f}} \phi \underline{v}_{\mathrm{f}}^{2} d \Omega,
$$

and the total Helmholtz free energy

$$
\mathcal{W}=\int_{\Omega^{0}} \Psi(\underline{\underline{e}}, m) d \Omega,
$$

we can now recall the following energy balance result, see [10, Theorem 7$]$

$$
\begin{array}{r}
\frac{d \mathcal{K}}{d t}+\frac{d \mathcal{W}}{d t}=-\int_{\Omega^{0}} \frac{\partial \Psi^{\text {damp }}}{\partial \underline{\underline{\dot{e}}}}: \underline{\underline{\dot{e}}} d \Omega-\int_{\Omega_{t}} \phi \underline{\underline{\sigma}}_{\mathrm{vis}}: \underline{\underline{\varepsilon}}\left(\underline{v}_{\mathrm{f}}\right) d \Omega-\int_{\Omega_{t}}\left(\underline{v}_{\mathrm{f}}-\underline{v}_{\mathrm{s}}\right) \cdot \phi^{2} \underline{\underline{k}}_{\mathrm{f}}^{-1} \cdot\left(\underline{v}_{\mathrm{f}}-\underline{v}_{\mathrm{s}}\right) d \Omega \\
+\mathcal{P}_{\text {ext }}^{\text {total }}+\mathcal{J}_{\mathcal{K} b}+\mathcal{J}_{\mathcal{K} \theta}+\mathcal{J}_{\mathcal{W} b}+\mathcal{J}_{\mathcal{G} \theta},
\end{array}
$$

the operator $\underline{\underline{\varepsilon}}$ denoting the usual symmetrized gradient, and with

$$
\mathcal{P}_{\text {ext }}^{\text {total }}=\int_{\Omega^{0}} \rho_{s 0}\left(1-\phi_{0}\right) \underline{f} \cdot \underline{v}_{\mathrm{s}} d \Omega+\int_{\Omega_{t}} \rho_{\mathrm{f}} \phi \underline{f} \cdot \underline{v}_{\mathrm{f}} d \Omega+\int_{\partial \Omega_{t}} \underline{t} \cdot\left((1-\phi) \underline{v}_{\mathrm{s}}+\phi \underline{v}_{\mathrm{f}}\right) d S
$$

the total power of external forces,

$$
\mathcal{J}_{\mathcal{K} b}=-\frac{1}{2} \int_{\partial \Omega_{t}} \rho_{\mathrm{f}} \phi \underline{v}_{\mathrm{f}}^{2}\left(\underline{v}_{\mathrm{f}}-\underline{v}_{\mathrm{s}}\right) \cdot \underline{n} d S, \quad \mathcal{J}_{\mathcal{K} \theta}=\frac{1}{2} \int_{\Omega_{t}} \underline{v}_{\mathrm{f}}^{2} \theta d \Omega
$$

the incoming rates of fluid kinetic energy due to the boundary flow and source term, respectively, and

$$
\mathcal{J}_{\mathcal{W} b}=-\int_{\partial \Omega_{t}} \rho_{\mathrm{f}} \phi \psi_{m}\left(\underline{v}_{\mathrm{f}}-\underline{v}_{\mathrm{s}}\right) \cdot \underline{n} d S, \quad \mathcal{J}_{\mathcal{G} \theta}=\int_{\Omega_{t}} g_{m} \theta d \Omega
$$

similar incoming rates of Helmholtz and Gibbs free energies. The physical interpretation of (5) is that total energy variations correspond to dissipation losses - in the fluid and solid phases separately, and in their interaction - and external source terms.

\section{Effective and energy-preserving time discretization}

In the sequel we use the standard mid-point notation

$$
g^{n+\frac{1}{2}}=\frac{g^{n}+g^{n+1}}{2},
$$


except when otherwise specified for some specific quantities that we then denote by $g^{n+\frac{1}{2} \sharp}$, to then emphasize that a discretization rule other than simple mid-point is being considered.

For any field $\underline{w}_{D}^{0}$ in $V^{0}=H^{1}\left(\Omega^{0}\right)^{3}$, we define

$$
V^{0}\left(\underline{w}_{D}^{0}\right)=\left\{\underline{v}^{*} \in V^{0}\left|\underline{v}^{*}\right|_{D}^{0}=\underline{w}_{D}^{0}\right\} .
$$

Assuming a sufficiently regular mapping between the reference domain $\Omega^{0}$ and the deformed configuration of time step $n$ denoted by $\Omega_{\mathrm{f}}^{n}$, we define

$$
Q^{n}=L^{2}\left(\Omega_{\mathrm{f}}^{n}\right),
$$

and for any $\left(\underline{w}_{D}, \underline{w}\right)$ in $\left(V^{n}\right)^{2}=\left(H^{1}\left(\Omega_{\mathrm{f}}^{n}\right)^{3}\right)^{2}$,

$$
\begin{aligned}
V^{n}\left(\underline{w}_{D}\right) & =\left\{\underline{v}^{*} \in V^{n}\left|\underline{v}^{*}\right|_{D}^{n}=\underline{w}_{D}\right\}, \\
V^{n}\left(\underline{w}_{D}, \underline{w}\right) & =\left\{\underline{v}^{*} \in V^{n}\left|\underline{v}^{*}\right|_{D}^{n}=\underline{w}_{D},\left.\underline{v}^{*}\right|_{\Gamma_{N \mathrm{nos}}^{n}}=\left.\underline{w}\right|_{\Gamma_{N \mathrm{nos}}^{n}},\left(\underline{v}^{*}-\underline{w}\right) \cdot \underline{n}=0 \text { on } \Gamma_{N \mathrm{nof}}^{n}\right\} .
\end{aligned}
$$

As in the continuous framework, we will use the same notation for functions defined in $\Omega^{0}$ and $\Omega_{\mathrm{f}}^{n}$.

All the solution spaces considered here are implicitly assumed from now on to be discrete in space, typically using a finite element type strategy, but we do not dwell on space discretization in this paper. Nevertheless, we will denote by $h$ the typical maximum diameter of all the finite elements in the mesh.

\subsection{Time-discrete partitioned coupling method}

We now define our proposed partitioned method, drawing some inspiration from the method previously proposed in [1] for fluid-structure interaction, see above Remark 1.

Given the solutions $\left(\underline{y}_{\mathrm{s}}^{n}, \underline{v}_{\mathrm{s}}^{n}\right)$ and $\left(\underline{v}_{\mathrm{f}}^{n}, m^{n}\right)$ up to time step $n$, perform the following steps:

- Step 0. Mesh and porosity updates: $\Omega_{\mathrm{f}}^{n+1}=\left(I_{\Omega^{0}}+\underline{y}_{\mathrm{s}}^{n}\right) \Omega^{0}$ and $\phi^{n}=\frac{m^{n} / \rho_{\mathrm{f}}+\phi_{0}}{J\left(\underline{y}_{\mathrm{s}}^{n}\right)}$.

- Step 1. Explicit step: find $\underline{\tilde{v}}_{\mathrm{f}}^{n+1} \in V^{n+1}\left(\underline{v}_{\mathrm{f}}^{\mathrm{pr}}\left(t^{n+1}\right)\right)$ such that $\forall \underline{\tilde{v}}_{\mathrm{f}}^{*} \in V^{n+1}(0)$

$$
\begin{aligned}
& \int_{\Omega_{\mathrm{f}}^{n+1}} \frac{\rho_{\mathrm{f}}}{\Delta t} \phi^{n} \underline{\tilde{v}}_{\mathrm{f}}^{n+1} \cdot \underline{\tilde{v}}_{\mathrm{f}}^{*} d \Omega-\int_{\Omega_{\mathrm{f}}^{n}} \frac{\rho_{\mathrm{f}}}{\Delta t} \phi^{n-1} \underline{v}_{\mathrm{f}}^{n} \cdot \underline{\tilde{v}}_{\mathrm{f}}^{*} d \Omega \\
& +\int_{\Omega_{\mathrm{f}}^{n}} \underline{\nabla}_{\underline{\mathrm{x}}} \cdot\left(\rho_{\mathrm{f}} \phi^{n-1} \underline{\underline{v}}_{\mathrm{f}}^{n+1} \otimes\left(\underline{v}_{\mathrm{f}}^{n}-\underline{v}_{\mathrm{s}}^{n-\frac{1}{2}}\right)\right) \cdot \underline{\tilde{v}}_{\mathrm{f}}^{*} d \Omega+2 \mu \int_{\Omega_{\mathrm{f}}^{n+1}} \phi^{n} \underline{\underline{\varepsilon}}\left(\underline{\tilde{v}}_{\mathrm{f}}^{n+1}\right): \underline{\varepsilon}_{\underline{\mathrm{\varepsilon}}}\left(\underline{\tilde{v}}_{\mathrm{f}}^{*}\right) d \Omega \\
& +\frac{\gamma \mu}{h} \int_{\Gamma_{N \text { nos }}^{n+1}} \phi^{n}\left(\underline{\tilde{v}}_{\mathrm{f}}^{n+1}-\underline{v}_{\mathrm{s}}^{n-\frac{1}{2}}\right) \cdot \underline{\tilde{v}}_{\mathrm{f}}^{*} d S+\frac{\gamma \mu}{h} \int_{\Gamma_{N \text { nof }}^{n+1}} \phi^{n}\left[\left(\underline{\tilde{v}}_{\mathrm{f}}^{n+1}-\underline{v}_{\mathrm{s}}^{n-\frac{1}{2}}\right) \cdot \underline{n}\right] \underline{\tilde{v}}_{\mathrm{f}}^{*} \cdot \underline{n} d S \\
& =2 \mu \int_{\Gamma_{N \text { nos }}^{n+1} \cup \Gamma_{N \text { nof }}^{n+1}} \phi^{n} \underline{\underline{\varepsilon}}\left(\underline{\tilde{v}}_{\mathrm{f}}^{n+1}\right) \cdot \underline{n} \cdot \underline{\tilde{v}}_{\mathrm{f}}^{*} d S+\int_{\Omega_{\mathrm{f}}^{n+1}} \theta^{n} \underline{\tilde{v}}_{\mathrm{f}}^{n+1} \cdot \underline{\tilde{v}}_{\mathrm{f}}^{*} d \Omega,
\end{aligned}
$$

where $\gamma$ denotes a stabilization parameter, see Remark 2 below.

- Step 2. Implicit step (implicit coupling of two sub-steps, fluid and solid)

- Step 2a. Fluid projection sub-step (where $\left.p^{n+1} \hat{=} \rho_{\mathrm{f}} \frac{\partial \Psi}{\partial m}\right|^{n+\frac{1}{2} \sharp}$, see below) 
Find $\left(\underline{v}_{\mathrm{f}}^{n+1}, m^{n+1}\right) \in V^{n+1}\left(\underline{v}_{\mathrm{f}}^{\mathrm{pr}}\left(t^{n+1}\right), \underline{v}_{\mathrm{s}}^{n+\frac{1}{2}}\right) \times Q^{n+1}$ such that $\forall\left(\underline{v}_{\mathrm{f}}^{*}, q^{*}\right) \in V^{n+1}(0,0) \times$ $Q^{n+1}$

$$
\left\{\begin{array}{c}
\int_{\Omega^{0}} \frac{m^{n+1}-m^{n}}{\Delta t} q^{*} d \Omega+\int_{\Omega_{\mathrm{f}}^{n+1}} \underline{\nabla}_{\underline{\mathrm{x}}} \cdot\left(\rho_{\mathrm{f}} \phi^{n}\left(\underline{v}_{\mathrm{f}}^{n+1}-\underline{v}_{\mathrm{s}}^{n+\frac{1}{2}}\right)\right) q^{*} d \Omega=\int_{\Omega_{\mathrm{f}}^{n+1}} q^{*} \theta^{n} d \Omega \\
\int_{\Omega_{\mathrm{f}}^{n+1}} \frac{\rho_{\mathrm{f}}}{\Delta t} \phi^{n}\left(\underline{v}_{\mathrm{f}}^{n+1}-\underline{v}_{\mathrm{f}}^{n+1}\right) \cdot \underline{v}_{\mathrm{f}}^{*} d \Omega-\int_{\Omega_{\mathrm{f}}^{n+1}} p^{n+1}\left(\underline{\nabla}_{\underline{\mathrm{x}}} \phi^{n}\right) \cdot \underline{v}_{\mathrm{f}}^{*} d \Omega \\
-\int_{\Omega_{\mathrm{f}}^{n+1}} \phi^{n} p^{n+1} \underline{\nabla}_{\mathrm{x}} \cdot \underline{v}_{\mathrm{f}}^{*} d \Omega+\int_{\Omega_{\mathrm{f}}^{n+1}}\left(\underline{v}_{\mathrm{f}}^{n+1}-\underline{v}_{\mathrm{s}}^{n+\frac{1}{2}}\right) \cdot\left|\phi^{n}\right|^{2} \underline{D}_{\mathrm{f}} \cdot \underline{v}_{\mathrm{f}}^{*} d \Omega \\
=\int_{\Omega_{\mathrm{f}}^{n+1}} \rho_{\mathrm{f}} \phi^{n} \underline{f}^{n+1} \cdot \underline{v}_{\mathrm{f}}^{*} d \Omega+\int_{\Gamma_{N}^{n+1} \cup \Gamma_{N \text { nof }}^{n+1}} \phi^{n} \underline{t}^{n+1} \cdot \underline{v}_{\mathrm{f}}^{*} d S
\end{array}\right.
$$

- Step 2b. Solid step (Newmark mid-point scheme)

Find $\left(\underline{y}_{\mathrm{s}}^{n+1}, \underline{v}_{\mathrm{s}}^{n+1}\right) \in V^{0}\left(\underline{y}_{\mathrm{s}}^{\mathrm{pr}}\left(t^{n+1}\right)\right) \times V^{0}\left(\underline{\dot{y}}_{\mathrm{s}}^{\mathrm{pr}}\left(t^{n+1}\right)\right)$ such that $\forall \underline{v}_{\mathrm{s}}^{*} \in V^{0}(0)$

$$
\left\{\begin{aligned}
\frac{\underline{y}_{\mathrm{s}}^{n+1}-\underline{y}_{\mathrm{s}}^{n}}{\Delta t}= & \frac{\underline{v}_{\mathrm{s}}^{n+1}+\underline{v}_{\mathrm{s}}^{n}}{2} \\
\int_{\Omega^{0}} \frac{\rho_{\mathrm{s} 0}}{\Delta t}\left(1-\phi_{0}\right)\left(\underline{v}_{\mathrm{s}}^{n+1}-\underline{v}_{\mathrm{s}}^{n}\right) \cdot \underline{v}_{\mathrm{s}}^{*} d \Omega & \\
+\int_{\Omega^{0}} & \left(\left.\frac{\partial \Psi}{\partial \underline{\underline{e}}}\right|^{n+\frac{1}{2} \sharp}+\left.\frac{\partial \Psi^{\mathrm{damp}}}{\partial \underline{\underline{e}}}\right|^{n+\frac{1}{2} \sharp}\right): d_{\underline{y}} \underline{\underline{e}}^{n+\frac{1}{2} \sharp} \cdot \underline{v}_{\mathrm{s}}^{*} d \Omega \\
& +\int_{\Omega_{\mathrm{f}}^{n+1}} \phi^{n} p^{n+1} \underline{\nabla}_{\underline{\mathrm{x}}} \cdot \underline{v}_{\mathrm{s}}^{*} d \Omega+\frac{\gamma \mu}{h} \int_{\Gamma_{N \text { nos }}^{n+1}} \phi^{n}\left(\underline{v}_{\mathrm{s}}^{n+\frac{1}{2}}-\underline{v}_{\mathrm{s}}^{n-\frac{1}{2}}\right) \cdot \underline{v}_{\mathrm{s}}^{*} d S \\
& +\frac{\gamma \mu}{h} \int_{\Gamma_{N \mathrm{nof}}^{n+1}} \phi^{n}\left[\left(\underline{v}_{\mathrm{s}}^{n+\frac{1}{2}}-\underline{v}_{\mathrm{s}}^{n-\frac{1}{2}}\right) \cdot \underline{n}\right] \underline{v}_{\mathrm{s}}^{*} \cdot \underline{n} d S \\
& =\int_{\Omega^{0}} \rho_{\mathrm{s} 0}\left(1-\phi_{0}\right) \underline{f}^{n+1} \cdot \underline{v}_{\mathrm{s}}^{*} d \Omega+\int_{\Gamma_{N}^{0}}\left(1-\phi^{n}\right) \underline{t}_{0}^{n+1} \cdot \underline{v}_{\mathrm{s}}^{*} d S \\
& +\int_{\Gamma_{N \mathrm{nos}}^{0} \cup \Gamma_{N \mathrm{nof}}^{0}} \underline{t}_{0}^{n+1} \cdot \underline{v}_{\mathrm{s}}^{*} d S-\int_{\Gamma_{N \mathrm{nof}}^{n+1}} \phi\left(\pi_{\tau} \underline{t}^{n+1}\right) \cdot \underline{v}_{\mathrm{s}}^{*} d S-\overline{\mathcal{R}}_{\mathrm{f}}^{d}\left(\underline{v}_{\mathrm{s}}^{*}\right)
\end{aligned}\right.
$$

Here, $\overline{\mathcal{R}}_{\mathrm{f}}^{d}$ denotes the discrete version of $\overline{\mathcal{R}}_{\mathrm{f}}^{c}$, such that for any $\underline{v}^{*}$ in $V^{n+1}$

$$
\begin{aligned}
\overline{\mathcal{R}}_{\mathrm{f}}^{d}\left(\underline{v}^{*}\right)= & \int_{\Omega_{\mathrm{f}}^{n+1}} \frac{\rho_{\mathrm{f}}}{\Delta t} \phi^{n} \underline{v}_{\mathrm{f}}^{n+1} \cdot \underline{v}^{*} d \Omega-\int_{\Omega_{\mathrm{f}}^{n}} \frac{\rho_{\mathrm{f}}}{\Delta t} \phi^{n-1} \underline{v}_{\mathrm{f}}^{n} \cdot \underline{v}^{*} d \Omega \\
& +\int_{\Omega_{\mathrm{f}}^{n}} \underline{\nabla}_{\underline{\mathrm{x}}} \cdot\left(\rho_{\mathrm{f}} \phi^{n-1} \underline{\underline{v}}_{\mathrm{f}}^{n+1} \otimes\left(\underline{v}_{\mathrm{f}}^{n}-\underline{v}_{\mathrm{s}}^{n-\frac{1}{2}}\right)\right) \cdot \underline{v}^{*} d \Omega-\int_{\Omega_{\mathrm{f}}^{n+1}} \theta^{n} \underline{\underline{v}}_{\mathrm{f}}^{n+1} \cdot \underline{v}^{*} d \Omega \\
& -\int_{\Omega_{\mathrm{f}}^{n+1}} \phi^{n} p^{n+1} \underline{\nabla_{\mathrm{x}}} \cdot \underline{v}^{*} d \Omega+2 \mu \int_{\Omega_{\mathrm{f}}^{n+1}} \phi^{n} \underline{\underline{\varepsilon}}\left(\underline{\tilde{v}}_{\mathrm{f}}^{n+1}\right): \underline{\underline{\varepsilon}}\left(\underline{v}^{*}\right) d \Omega \\
& -\int_{\Omega_{\mathrm{f}}^{n+1}} \rho_{\mathrm{f}} \phi^{n} \underline{f}^{n+1} \cdot \underline{v}^{*} d \Omega-\int_{\Gamma_{N}^{n+1}} \phi^{n} \underline{t}^{n+1} \cdot \underline{v}^{*} d S-\int_{\Gamma_{N \text { nof }}^{n+1}} \phi^{n}\left(\pi_{\tau} \underline{t}^{n+1}\right) \cdot \underline{v}^{*} d S,
\end{aligned}
$$

the strain-related quantities are discretized as follows

$$
\begin{gathered}
\underline{\underline{e}}^{n+\frac{1}{2} \sharp}=\underline{\underline{e}}\left(\underline{y}_{\mathrm{s}}^{n+\frac{1}{2}}\right), \quad \underline{\underline{e}}^{n+\frac{1}{2} \sharp}=\frac{\underline{\underline{e}}^{n+1}-\underline{\underline{e}}^{n}}{\Delta t}, \\
d_{\underline{y}} \underline{\underline{e}}^{n+\frac{1}{2} \sharp} \cdot \underline{v}^{*}=\frac{1}{2}\left(\underline{\underline{F}}\left(\underline{y}_{\mathrm{s}}^{n+\frac{1}{2}}\right)^{T} \cdot \underline{\underline{\nabla}}_{\underline{\xi}} \underline{v}^{*}+\underline{\underline{\nabla}}_{\underline{\xi}}^{T} \underline{v}^{*} \cdot \underline{\underline{F}}\left(\underline{y}_{\mathrm{s}}^{n+\frac{1}{2}}\right)\right),
\end{gathered}
$$


and the discrete solid stress tensor contribution is obtained using (4), with the following discretization choices for $\left.\frac{\partial \Psi}{\partial \underline{\underline{e}}}\right|^{n+\frac{1}{2} \sharp}$ (see $\left.[22]\right)$ and $p^{n+1}$

$$
\left\{\begin{array}{l}
\left.\frac{\partial \Psi}{\partial \underline{\underline{e}}}\right|^{n+\frac{1}{2} \sharp} \hat{=} \frac{\partial \Psi}{\partial \underline{\underline{e}}}\left(\underline{\underline{e}}^{n+\frac{1}{2} \sharp}, m^{n+1}\right) \\
\quad+\left(\frac{\Psi\left(\underline{\underline{e}}^{n+1}, m^{n+1}\right)-\Psi\left(\underline{\underline{e}}^{n}, m^{n+1}\right)}{\Delta t}-\frac{\partial \Psi}{\partial \underline{\underline{e}}}\left(\underline{\underline{e}}^{n+\frac{1}{2} \sharp}, m^{n+1}\right): \underline{\underline{e}}^{n+\frac{1}{2}}\right) \frac{\underline{\underline{e}}^{n+\frac{1}{2}}}{\underline{\underline{e}}^{n+\frac{1}{2}}: \underline{\underline{e}}^{n+\frac{1}{2}}} \\
\left.\frac{\underline{p}^{n+1}}{\rho_{\mathrm{f}}} \hat{=} \frac{\partial \Psi}{\partial m}\right|^{n+\frac{1}{2} \sharp} \hat{=} \frac{\Psi\left(\underline{\underline{e}}^{n}, m^{n+1}\right)-\Psi\left(\underline{\underline{e}}^{n}, m^{n}\right)}{m^{n+1}-m^{n}} .
\end{array}\right.
$$

In the sequel, we will choose $\Psi^{\text {damp }}=\frac{\eta_{\mathrm{d}}}{2} \operatorname{tr}(\underline{\underline{e}})^{2}$, with the discretization

$$
\left.\int_{\Omega^{0}} \frac{\partial \Psi^{\mathrm{damp}}}{\partial \underline{\underline{\dot{e}}}}\right|^{n+\frac{1}{2} \sharp}: d_{\underline{y}} \underline{e}^{n+\frac{1}{2} \sharp} \cdot \underline{v}_{\mathrm{S}}^{*} d \Omega=\int_{\Omega^{0}} \eta_{\mathrm{d}} d_{\underline{y}} \underline{\underline{e}}^{n+\frac{1}{2} \sharp} \cdot \underline{v}_{\mathrm{s}}^{n+\frac{1}{2}}: d_{\underline{y}} \underline{e}^{n+\frac{1}{2} \sharp} \cdot \underline{v}_{\mathrm{s}}^{*} d \Omega .
$$

\section{Remark 2 (Splitting and Robin boundary conditions rationale)}

In essence, Step 1 takes care of the advection-diffusion part of the fluid problem, with an explicit treatment of the advection term (linear problem), and no coupling with the solid part other than via the Robin-type boundary conditions on the "fluid-proof" boundaries, namely, $\Gamma_{N \text { nof }}^{0} \cup \Gamma_{N \text { nos }}^{0}$. Then, Step 2 corrects the velocity computed in Step 1 by taking into account the coupling of the solid component with the pressure part of the fluid problem, including for the distributed friction term. Eventually, the fluid inertia term $\int_{\Omega^{0}} \frac{\mathrm{d}}{\mathrm{dt}}\left(J \rho_{\mathrm{f}} \phi \underline{v}_{\mathrm{f}}\right) \cdot \underline{v}^{*} d \Omega$ of the continuous problem is decomposed in the discrete problem into two contributions that appear in Steps 1 and 2a, i.e., respectively,

$$
\int_{\Omega_{\mathrm{f}}^{n+1}} \frac{\rho_{\mathrm{f}}}{\Delta t} \phi^{n} \underline{\tilde{v}}_{\mathrm{f}}^{n+1} \cdot \underline{v}^{*} d \Omega-\int_{\Omega_{\mathrm{f}}^{n}} \frac{\rho_{\mathrm{f}}}{\Delta t} \phi^{n-1} \underline{v}_{\mathrm{f}}^{n} \cdot \underline{v}^{*} d \Omega \quad \text { and } \quad \int_{\Omega_{\mathrm{f}}^{n+1}} \rho_{\mathrm{f}} \phi^{n} \frac{\underline{v}_{\mathrm{f}}^{n+1}-\underline{\tilde{v}}_{\mathrm{f}}^{n+1}}{\Delta t} \cdot \underline{v}^{*} d \Omega .
$$

As for Robin boundary conditions, they are introduced in Steps 1 and $2 \mathrm{~b}$ - with the associated stabilization parameter $\gamma$ - to take care of Dirichlet boundary conditions that relate the fluid and solid velocities, while ensuring stability in the coupling, as will be demonstrated in the below stability analysis. This combination of splitting strategy with Robin boundary conditions is similar to that proposed in [1] for fluid-structure interaction, albeit here extended to a more complex problem.

\subsection{Stability analysis}

Our objective in this section is to establish the stability of our proposed scheme, namely, a discrete energy balance similar to (5) up to some numerical dissipation terms. We will then naturally assume that there exists a solution $\left(\underline{y}_{\mathrm{s}}^{n}, \underline{v}_{\mathrm{s}}^{n}, \underline{v}_{\mathrm{f}}^{n}, m^{n}\right)$ to the discrete equations (6)-(8) up to time step $n$, and that this solution is admissible, which we characterize by

$$
J\left(\underline{y}_{\mathrm{s}}^{n}\right)>0, \quad 0<\phi^{n}=\frac{m^{n} / \rho_{\mathrm{f}}+\phi_{0}}{J\left(\underline{y}_{\mathrm{s}}^{n}\right)}<1,
$$

everywhere, with obvious physical interpretations for these conditions. In fact, in the stability analysis, for technical reasons we will make a stronger assumption on the jacobian $J\left(\underline{y}_{\mathrm{s}}^{n}\right)$, i.e.

$$
\frac{\max _{\xi \in \Omega^{0}} J\left(\underline{y}_{\mathrm{s}}^{n}\right)}{\min _{\xi \in \Omega^{0}} J\left(\underline{y}_{\mathrm{s}}^{n}\right)}<M,
$$

with $M$ independent of $n$. This is in order to be able to invoke the following inverse inequality. 


\section{Lemma 1}

Assuming that (10) holds, there exists a constant $C_{i e}$ such that

$$
\left\|\sqrt{\phi^{n}} \underline{\underline{\varepsilon}}\left(\underline{v}^{*}\right) \cdot \underline{n}\right\|_{L^{2}\left(\Gamma_{\text {Nnos }}^{n+1}\right)}^{2} \leq \frac{C_{i e}}{h}\left\|\sqrt{\phi^{n}} \underline{\underline{\varepsilon}}\left(\underline{v}^{*}\right)\right\|_{L^{2}\left(\Omega_{\mathrm{f}}^{n+1}\right)}^{2}, \quad \forall \underline{v}^{*} \in H^{1}\left(\Omega_{\mathrm{f}}^{n}\right)^{3} .
$$

This inverse inequality is obtained by a standard scaling argument when noting that

$$
\left\|\sqrt{\phi^{n}} \underline{\underline{\varepsilon}}\left(\underline{v}^{*}\right) \cdot \underline{n}\right\|_{L^{2}\left(\Gamma_{N \mathrm{nos}}^{n+1}\right)}^{2}=\left\|\phi^{n}\left(\underline{\underline{\varepsilon}}\left(\underline{v}^{*}\right) \cdot \underline{n}\right)^{2}\right\|_{L^{1}\left(\Gamma_{N \mathrm{nos}}^{n+1}\right)}=\left\|\frac{m^{n} / \rho_{\mathrm{f}}+\phi_{0}}{J\left(\underline{y}_{\mathrm{s}}^{n}\right)}\left(\underline{\underline{\varepsilon}}\left(\underline{v}^{*}\right) \cdot \underline{n}\right)^{2}\right\|_{L^{1}\left(\Gamma_{N \mathrm{nos}}^{n+1}\right)},
$$

where $\left(m^{n} / \rho_{\mathrm{f}}+\phi_{0}\right) \underline{\underline{\varepsilon}}\left(\underline{v}^{*}\right)^{2}$ is a polynomial - hence, in a finite dimensional space - due to the spatial discretization of $m^{n}$ and $\underline{v}^{*}$. By contrast, $1 / J\left(\underline{y}_{\mathrm{s}}^{n}\right)$ is not a polynomial in general, which leads to Condition (10).

Remark 3 (Inverse inequality and condition (10))

First of all, it should be noted that the inverse inequality (11) is rather "conservative" in itself, due to the fact that only the mesh elements adjacent to the boundary $\Gamma_{N \text { nos }}^{n+1}$ are concerned in the left-hand side, hence, in the starting point of the scaling argument. Moreover, in the course of the scaling argument, Condition (10) could clearly be relaxed in the form

$$
\frac{\max _{\xi \in K} J\left(\underline{y}_{\mathrm{s}}^{n}\right)}{\min _{\xi \in K} J\left(\underline{y}_{\mathrm{s}}^{n}\right)}<M,
$$

for every finite element $K$ in the mesh. A particular case arises when considering linear finite elements for the solid displacements, in which case the Jacobian is constant within each element.

For the sake of simplicity in the stability analysis, we will assume that $\underline{t}=0$ on $\Gamma_{N \text { nos }}$, $\Gamma_{N \text { nof }}=\varnothing, \underline{f}=\underline{0}, \theta=0, \underline{y}_{\mathrm{s}}^{\mathrm{pr}}=\underline{0}$ and $\underline{v}_{\mathrm{f}}^{\mathrm{pr}}=\underline{0}$. We then have the following result.

\section{Proposition 2}

Assuming that (10) holds and that $\gamma>C_{i e}$, the time scheme (6)-(8) satisfies

$$
\begin{aligned}
& \frac{\mathcal{E}^{n+1}-\mathcal{E}^{n}}{\Delta t}+\frac{\gamma \mu}{2 h}\left\|\underline{v}_{\mathrm{s}}^{n+\frac{1}{2}}\right\|_{\phi^{n}, \Gamma_{N \text { nos }}^{n+1}}^{2}-\frac{\gamma \mu}{2 h}\left\|\underline{v}_{\mathrm{s}}^{n-\frac{1}{2}}\right\|_{\phi^{n}, \Gamma_{N \text { nos }}^{n+1}}^{2} \\
& \leq\left(\underline{t}^{n+1}, \underline{v}_{\mathrm{f}}^{n+1}\right)_{\phi^{n}, \Gamma_{N}^{n+1}}+\left(\underline{t}_{0}^{n+1}, \underline{v}_{\mathrm{s}}^{n+\frac{1}{2}}\right)_{1-\phi^{n}, \Gamma_{N}^{0}}-\left.\int_{\Omega^{0}} \frac{\partial \Psi^{d a m p}}{\partial \underline{\underline{\dot{e}}}}\right|^{n+\frac{1}{2} \sharp}: d_{\underline{y}} \underline{\underline{e}}^{n+\frac{1}{2} \sharp} \cdot \underline{v}_{\mathrm{s}}^{n+\frac{1}{2}} d \Omega \\
& -\int_{\Omega_{\mathrm{f}}^{n+1}}\left(\underline{v}_{\mathrm{f}}^{n+1}-\underline{v}_{\mathrm{s}}^{n+\frac{1}{2}}\right) \cdot\left|\phi^{n}\right|^{2} \underline{\underline{D}}_{\mathrm{f}} \cdot\left(\underline{v}_{\mathrm{f}}^{n+1}-\underline{v}_{\mathrm{s}}^{n+\frac{1}{2}}\right) d \Omega-\mathcal{T}_{1} \\
& -\frac{\rho_{\mathrm{f}}}{2 \Delta t}\left\|\underline{\tilde{v}}_{\mathrm{f}}^{n+1}-\underline{v}_{\mathrm{f}}^{n}\right\|_{\phi^{n-1}, \Omega_{\mathrm{f}}^{n}}^{2}-\frac{\rho_{\mathrm{f}}}{2 \Delta t}\left\|\underline{v}_{\mathrm{f}}^{n+1}-\underline{\tilde{v}}_{\mathrm{f}}^{n+1}\right\|_{\phi^{n}, \Omega_{\mathrm{f}}^{n+1}}^{2} \\
& -C \mu\left\|\underline{\underline{\varepsilon}}\left(\underline{\tilde{v}}_{\mathrm{f}}^{n+1}\right)\right\|_{\phi^{n}, \Omega_{\mathrm{f}}^{n+1}}^{2}-C \frac{\mu}{L}\left\|\underline{\tilde{v}}_{\mathrm{f}}^{n+1}-\underline{v}_{\mathrm{s}}^{n+\frac{1}{2}}\right\|_{\phi^{n}, \Gamma_{N \text { nos }}^{n+1}}^{2},
\end{aligned}
$$

with $C$ a positive dimensionless constant, $L$ homogeneous to a length, $\mathcal{E}^{n}$ the total discrete energy at step $n$, i.e.

$$
\mathcal{E}^{n}=\mathcal{K}_{\mathrm{f}}^{n}+\mathcal{K}_{\mathrm{s}}^{n}+\mathcal{W}^{n}=\frac{\rho_{\mathrm{f}}}{2}\left\|\underline{v}_{\mathrm{f}}^{n}\right\|_{\phi^{n-1}, \Omega_{\mathrm{f}}^{n}}^{2}+\frac{\rho_{\mathrm{s} 0}}{2}\left\|\underline{v}_{\mathrm{s}}^{n}\right\|_{1-\phi_{0}, \Omega^{0}}^{2}+\int_{\Omega^{0}} \Psi\left(\underline{\underline{e}}^{n}, m^{n}\right) d \Omega,
$$

and $\mathcal{T}_{1}$ the discrete flux of outgoing fluid kinetic energy that crosses the domain borders

$$
\mathcal{T}_{1}=\frac{1}{2} \int_{\partial \Omega_{\mathrm{f}}^{n} \backslash \Gamma_{N n o s}^{n}} \rho_{\mathrm{f}} \phi^{n-1}\left|\underline{\tilde{v}}_{\mathrm{f}}^{n+1}\right|^{2}\left(\underline{v}_{\mathrm{f}}^{n}-\underline{v}_{\mathrm{s}}^{n-\frac{1}{2}}\right) \cdot \underline{n} d S .
$$


Proof. Respectively evaluating the equations (6), (7b) and (8) with the test functions

$$
\underline{\tilde{v}}_{\mathrm{f}}^{*}=\underline{\tilde{v}}_{\mathrm{f}}^{n+1}, \quad \underline{v}_{\mathrm{f}}^{*}=\underline{v}_{\mathrm{f}}^{n+1}-\underline{v}_{\mathrm{s}}^{n+\frac{1}{2}}, \quad \text { and } \quad \underline{v}_{\mathrm{s}}^{*}=\underline{v}_{\mathrm{s}}^{n+\frac{1}{2}},
$$

we get, defining

$$
\mathcal{R}_{p}\left(\underline{v}^{*}\right)=\int_{\Omega_{\mathrm{f}}^{n+1}} \rho_{\mathrm{f}} \phi^{n} \frac{\underline{v}_{\mathrm{f}}^{n+1}-\underline{\tilde{v}}_{\mathrm{f}}^{n+1}}{\Delta t} \cdot \underline{v}^{*} d \Omega-\int_{\Omega_{\mathrm{f}}^{n+1}} \phi^{n} p^{n+1} \underline{\nabla}_{\underline{\mathrm{x}}} \cdot \underline{v}^{*} d \Omega-\int_{\Gamma_{N}^{n+1}} \phi^{n} \underline{t}^{n+1} \cdot \underline{v}^{*} d S
$$

and using the weighted $L^{2}$-scalar product notation $(g, h)_{\psi, \Omega}=\int_{\Omega} \psi g h d \Omega$,

$$
\begin{aligned}
& \frac{\rho_{\mathrm{f}}}{\Delta t}\left(\underline{\tilde{v}}_{\mathrm{f}}^{n+1}, \underline{\tilde{v}}_{\mathrm{f}}^{n+1}\right)_{\phi^{n}, \Omega_{\mathrm{f}}^{n+1}}-\frac{\rho_{\mathrm{f}}}{\Delta t}\left(\underline{v}_{\mathrm{f}}^{n}, \underline{\tilde{v}}_{\mathrm{f}}^{n+1}\right)_{\phi^{n-1}, \Omega_{\mathrm{f}}^{n}} \\
& +\int_{\Omega_{\mathrm{f}}^{n}} \underline{\nabla}_{\underline{\mathrm{x}}} \cdot\left(\rho_{\mathrm{f}} \phi^{n-1} \underline{\tilde{v}}_{\mathrm{f}}^{n+1} \otimes\left(\underline{v}_{\mathrm{f}}^{n}-\underline{v}_{\mathrm{s}}^{n-\frac{1}{2}}\right)\right) \cdot \underline{\tilde{v}}_{\mathrm{f}}^{n+1} d \Omega+2 \mu\left\|\underline{\underline{\varepsilon}}\left(\underline{\tilde{v}}_{\mathrm{f}}^{n+1}\right)\right\|_{\phi^{n}, \Omega_{\mathrm{f}}^{n+1}} \\
& \left.+\frac{\gamma \mu}{h}\left(\underline{\tilde{v}}_{\mathrm{f}}^{n+1}-\underline{v}_{\mathrm{s}}^{n-\frac{1}{2}}, \underline{\tilde{v}}_{\mathrm{f}}^{n+1}\right)_{\phi^{n}, \Gamma_{N \text { nos }}^{n+1}}=2 \mu\left(\underline{\underline{\varepsilon}}_{\underline{\mathrm{f}}}^{n+1}\right) \cdot \underline{n}, \underline{\tilde{v}}_{\mathrm{f}}^{n+1}\right)_{\phi^{n}, \Gamma_{N \text { nos }}^{n+1}}, \\
& \frac{\rho_{\mathrm{f}}}{\Delta t}\left(\underline{v}_{\mathrm{f}}^{n+1}-\underline{\tilde{v}}_{\mathrm{f}}^{n+1}, \underline{v}_{\mathrm{f}}^{n+1}\right)_{\phi^{n}, \Omega_{\mathrm{f}}^{n+1}}-\int_{\Omega_{\mathrm{f}}^{n+1}} p^{n+1}\left(\underline{\nabla}_{\underline{\mathbf{x}}} \phi^{n}\right) \cdot\left(\underline{v}_{\mathrm{f}}^{n+1}-\underline{v}_{\mathrm{s}}^{n+\frac{1}{2}}\right) d \Omega \\
& -\int_{\Omega_{\mathrm{f}}^{n+1}} \phi^{n} p^{n+1} \underline{\nabla}_{\underline{\mathrm{x}}} \cdot \underline{v}_{\mathrm{f}}^{n+1} d \Omega+\int_{\Omega_{\mathrm{f}}^{n+1}}\left(\underline{v}_{\mathrm{f}}^{n+1}-\underline{v}_{\mathrm{s}}^{n+\frac{1}{2}}\right) \cdot\left|\phi^{n}\right|^{2} \underline{\underline{D}}_{\mathrm{f}} \cdot\left(\underline{v}_{\mathrm{f}}^{n+1}-\underline{v}_{\mathrm{s}}^{n+\frac{1}{2}}\right) d \Omega \\
& =\left(\underline{t}^{n+1}, \underline{v}_{\mathrm{f}}^{n+1}\right)_{\phi^{n}, \Gamma_{N}^{n+1}}+\mathcal{R}_{p}\left(\underline{v}_{\mathrm{s}}^{n+\frac{1}{2}}\right), \\
& \frac{\rho_{\mathrm{s} 0}}{2 \Delta t}\left\|\underline{v}_{\mathrm{s}}^{n+1}\right\|_{1-\phi_{0}, \Omega^{0}}^{2}-\frac{\rho_{\mathrm{s} 0}}{2 \Delta t}\left\|\underline{v}_{\mathrm{s}}^{n}\right\|_{1-\phi_{0}, \Omega^{0}}^{2} \\
& +\int_{\Omega^{0}}\left(\left.\frac{\partial \Psi}{\partial \underline{\underline{e}}}\right|^{n+\frac{1}{2} \sharp}+\left.\frac{\partial \Psi^{\mathrm{damp}}}{\partial \underline{\underline{\dot{e}}}}\right|^{n+\frac{1}{2} \sharp}\right): d_{\underline{y}} \underline{\underline{e}}^{n+\frac{1}{2} \sharp} \cdot \underline{v}_{\mathrm{s}}^{n+\frac{1}{2}} d \Omega+\int_{\Omega_{\mathrm{f}}^{n+1}} \phi^{n} p^{n+1} \underline{\nabla}_{\underline{\mathrm{x}}} \cdot \underline{v}_{\mathrm{s}}^{n+\frac{1}{2}} d \Omega \\
& +\frac{\gamma \mu}{h}\left(\underline{v}_{\mathrm{s}}^{n+\frac{1}{2}}-\underline{v}_{\mathrm{s}}^{n-\frac{1}{2}}, \underline{v}_{\mathrm{s}}^{n+\frac{1}{2}}\right)_{\phi^{n}, \Gamma_{N \text { nos }}^{n+1}}=\left(\underline{t}_{0}^{n+1} \cdot \underline{v}_{\mathrm{s}}^{n+\frac{1}{2}}\right)_{1-\phi^{n}, \Gamma_{N}^{0}}-\overline{\mathcal{R}}_{\mathrm{f}}^{d}\left(\underline{v}_{\mathrm{s}}^{n+\frac{1}{2}}\right) .
\end{aligned}
$$

For the first two terms of (13a), we use $2(a, b)=a^{2}+b^{2}-(a-b)^{2}$ to obtain

$$
\begin{aligned}
\frac{\rho_{\mathrm{f}}}{\Delta t} & \left(\underline{\tilde{v}}_{\mathrm{f}}^{n+1}, \underline{\tilde{v}}_{\mathrm{f}}^{n+1}\right)_{\phi^{n}, \Omega_{\mathrm{f}}^{n+1}}-\frac{\rho_{\mathrm{f}}}{\Delta t}\left(\underline{v}_{\mathrm{f}}^{n}, \underline{\tilde{v}}_{\mathrm{f}}^{n+1}\right)_{\phi^{n-1}, \Omega_{\mathrm{f}}^{n}} \\
& =\frac{\rho_{\mathrm{f}}}{\Delta t}\left(\left\|\underline{\tilde{v}}_{\mathrm{f}}^{n+1}\right\|_{\phi^{n}, \Omega_{\mathrm{f}}^{n+1}}^{2}-\frac{1}{2}\left\|\underline{\tilde{v}}_{\mathrm{f}}^{n+1}\right\|_{\phi^{n-1}, \Omega_{\mathrm{f}}^{n}}^{2}-\frac{1}{2}\left\|\underline{v}_{\mathrm{f}}^{n}\right\|_{\phi^{n-1}, \Omega_{\mathrm{f}}^{n}}^{2}+\frac{1}{2}\left\|\underline{\tilde{v}}_{\mathrm{f}}^{n+1}-\underline{v}_{\mathrm{f}}^{n}\right\|_{\phi^{n-1}, \Omega_{\mathrm{f}}^{n}}^{2}\right) .
\end{aligned}
$$

Then, the next term in (13a) can be rewritten as in the proof of [10, Theorem 7$]$

$$
\begin{aligned}
\int_{\Omega_{\mathrm{f}}^{n}} \underline{\nabla}_{\underline{\mathrm{x}}} \cdot\left(\rho_{\mathrm{f}} \phi^{n-1} \underline{\tilde{v}}_{\mathrm{f}}^{n+1} \otimes\left(\underline{v}_{\mathrm{f}}^{n}-\underline{v}_{\mathrm{s}}^{n-\frac{1}{2}}\right)\right) & \cdot \underline{\tilde{v}}_{\mathrm{f}}^{n+1} d \Omega \\
& =\int_{\Omega_{\mathrm{f}}^{n}} \frac{1}{2}\left|\underline{\tilde{v}}_{\mathrm{f}}^{n+1}\right|^{2} \underline{\nabla}_{\underline{\mathrm{x}}} \cdot\left(\rho_{\mathrm{f}} \phi^{n-1}\left(\underline{v}_{\mathrm{f}}^{n}-\underline{v}_{\mathrm{s}}^{n-\frac{1}{2}}\right)\right) d \Omega+\mathcal{T}_{1} .
\end{aligned}
$$


Now, recalling $\phi^{n}=\frac{m^{n} / \rho_{\mathrm{f}}+\phi_{0}}{J\left(\underline{y}_{\mathrm{s}}^{n}\right)}$ we evaluate (7a) at time step $n-1$ with $q^{*}=\left|\underline{\tilde{v}}_{\mathrm{f}}^{n+1}\right|^{2}-$ assuming this is allowed by the spatial discretization, see Section 3.3. This gives

$$
\begin{aligned}
& \frac{1}{2} \int_{\Omega_{\mathrm{f}}^{n}}\left|\underline{\tilde{v}}_{\mathrm{f}}^{n+1}\right|^{2} \underline{\nabla}_{\underline{\mathrm{x}}} \cdot\left(\rho_{\mathrm{f}} \phi^{n-1}\left(\underline{v}_{\mathrm{f}}^{n}-\underline{v}_{\mathrm{s}}^{n-\frac{1}{2}}\right)\right) d \Omega=-\frac{1}{2} \int_{\Omega^{0}} \frac{m^{n}-m^{n-1}}{\Delta t}\left|\underline{\tilde{v}}_{\mathrm{f}}^{n+1}\right|^{2} d \Omega \\
&=-\frac{1}{2} \int_{\Omega^{0}} \frac{\phi^{n} \rho_{\mathrm{f}} J\left(\underline{y}_{\mathrm{s}}^{n}\right)-\phi^{n-1} \rho_{\mathrm{f}} J\left(\underline{y}_{\mathrm{s}}^{n-1}\right)}{\Delta t}\left|\underline{\tilde{v}}_{\mathrm{f}}^{n+1}\right|^{2} d \Omega \\
&=-\frac{\rho_{\mathrm{f}}}{2 \Delta t}\left(\left\|\underline{\tilde{v}}_{\mathrm{f}}^{n+1}\right\|_{\phi^{n}, \Omega_{\mathrm{f}}^{n+1}}^{2}\left\|\underline{\tilde{v}}_{\mathrm{f}}^{n+1}\right\|_{\phi^{n-1}, \Omega_{\mathrm{f}}^{n}}^{2}\right) .
\end{aligned}
$$

Gathering (14), (15) and (16), we get

$$
\begin{array}{r}
\frac{\rho_{\mathrm{f}}}{\Delta t}\left(\underline{\tilde{v}}_{\mathrm{f}}^{n+1}, \underline{\tilde{v}}_{\mathrm{f}}^{n+1}\right)_{\phi^{n}, \Omega_{\mathrm{f}}^{n+1}}-\frac{\rho_{\mathrm{f}}}{\Delta t}\left(\underline{v}_{\mathrm{f}}^{n} \cdot \underline{\tilde{v}}_{\mathrm{f}}^{n+1}\right)_{\phi^{n-1}, \Omega_{\mathrm{f}}^{n}}+\int_{\Omega_{\mathrm{f}}^{n}} \underline{\nabla}_{\mathrm{x}} \cdot\left(\rho_{\mathrm{f}} \phi^{n-1} \underline{\tilde{v}}_{\mathrm{f}}^{n+1} \otimes\left(\underline{v}_{\mathrm{f}}^{n}-\underline{v}_{\mathrm{s}}^{n-\frac{1}{2}}\right)\right) \cdot \underline{\tilde{v}}_{\mathrm{f}}^{n+1} d \Omega \\
=\frac{\rho_{\mathrm{f}}}{2 \Delta t}\left(\left\|\underline{\tilde{v}}_{\mathrm{f}}^{n+1}\right\|_{\phi^{n}, \Omega_{\mathrm{f}}^{n+1}}^{2}-\left\|\underline{v}_{\mathrm{f}}^{n}\right\|_{\phi^{n-1}, \Omega_{\mathrm{f}}^{n}}^{2}+\left\|\underline{\tilde{v}}_{\mathrm{f}}^{n+1}-\underline{v}_{\mathrm{f}}^{n}\right\|_{\phi^{n-1}, \Omega_{\mathrm{f}}^{n}}^{2}\right)+\mathcal{T}_{1} .
\end{array}
$$

Then, substituting this result in (13a) and summing with (13b) and (13c) while applying the identity $(a-b, a)=\frac{1}{2} a^{2}-\frac{1}{2} b^{2}+\frac{1}{2}(a-b)^{2}$ to $\left(\underline{v}_{\mathrm{f}}^{n+1}-\underline{\tilde{v}}_{\mathrm{f}}^{n+1}, \underline{v}_{\mathrm{f}}^{n+1}\right)_{\phi^{n}, \Omega^{n+1}}$, we get (using $\left.\Delta t \underline{v}_{\mathrm{s}}^{n+\frac{1}{2}}=\underline{y}_{\mathrm{s}}^{n+1}-\underline{y}_{\mathrm{s}}^{n}\right)$

$$
\begin{aligned}
& \frac{\mathcal{K}_{\mathrm{f}}^{n+1}-\mathcal{K}_{\mathrm{f}}^{n}}{\Delta t}+\frac{\mathcal{K}_{\mathrm{s}}^{n+1}-\mathcal{K}_{\mathrm{s}}^{n}}{\Delta t}+\int_{\Omega^{0}}\left(\left.\frac{\partial \Psi}{\partial \underline{\underline{e}}}\right|^{n+\frac{1}{2} \sharp}+\left.\frac{\partial \Psi^{\mathrm{damp}}}{\partial \underline{\underline{e}}}\right|^{n+\frac{1}{2} \sharp}\right): d_{\underline{y}} \underline{\underline{e}}^{n+\frac{1}{2} \sharp} \cdot \underline{v}_{\mathrm{s}}^{n+\frac{1}{2}} d \Omega \\
& -\int_{\Omega_{\mathrm{f}}^{n+1}} \phi^{n} p^{n+1} \underline{\nabla}_{\underline{\mathrm{x}}} \cdot\left(\underline{v}_{\mathrm{f}}^{n+1}-\underline{v}_{\mathrm{s}}^{n+\frac{1}{2}}\right) d \Omega-\int_{\Omega_{\mathrm{f}}^{n+1}} p^{n+1}\left(\underline{\nabla}_{\underline{\mathbf{x}}} \phi^{n}\right) \cdot\left(\underline{v}_{\mathrm{f}}^{n+1}-\underline{v}_{\mathrm{s}}^{n+\frac{1}{2}}\right) d \Omega \\
& +\underbrace{\frac{\gamma \mu}{h}\left[\left(\underline{v}_{\mathrm{s}}^{n+\frac{1}{2}}-\underline{\tilde{v}}_{\mathrm{f}}^{n+1}, \underline{v}_{\mathrm{s}}^{n+\frac{1}{2}}\right)_{\phi^{n}, \Gamma_{N \text { nos }}^{n+1}}+\left(\underline{\tilde{v}}_{\mathrm{f}}^{n+1}-\underline{v}_{\mathrm{s}}^{n-\frac{1}{2}}, \underline{\tilde{v}}_{\mathrm{f}}^{n+1}\right)_{\left.\phi^{n}, \Gamma_{N \text { nos }}^{n+1}\right]}\right]}_{\mathcal{T}_{3}} \\
& =\left(\underline{t}^{n+1}, \underline{v}_{\mathrm{f}}^{n+1}\right)_{\phi^{n}, \Gamma_{N}^{n+1}}+\left(\underline{t}_{0}^{n+1}, \underline{v}_{\mathrm{s}}^{n+\frac{1}{2}}\right)_{1-\phi^{n}, \Gamma_{N}^{0}}-\mathcal{T}_{1} \\
& -2 \mu\left\|\underline{\underline{\varepsilon}}\left(\underline{\tilde{v}}_{\mathrm{f}}^{n+1}\right)\right\|_{\phi^{n}, \Omega_{\mathrm{f}}^{n+1}}^{2}-\int_{\Omega_{\mathrm{f}}^{n+1}}\left(\underline{v}_{\mathrm{f}}^{n+1}-\underline{v}_{\mathrm{s}}^{n+\frac{1}{2}}\right) \cdot\left|\phi^{n}\right|^{2} \underline{\underline{D}}_{\mathrm{f}} \cdot\left(\underline{v}_{\mathrm{f}}^{n+1}-\underline{v}_{\mathrm{s}}^{n+\frac{1}{2}}\right) d \Omega .
\end{aligned}
$$

We used here the following identity that comes from (6) evaluated with $\underline{\tilde{v}}_{\mathrm{f}}^{*}=\underline{v}_{\mathrm{s}}^{n+\frac{1}{2}}$

$$
\begin{aligned}
\overline{\mathcal{R}}_{\mathrm{f}}^{d}\left(\underline{v}_{\mathrm{s}}^{n+\frac{1}{2}}\right)-\mathcal{R}_{p}\left(\underline{v}_{\mathrm{s}}^{n+\frac{1}{2}}\right) & =2 \mu\left(\underline{\varepsilon}\left(\underline{v}_{\mathrm{f}}^{n+1}\right) \cdot \underline{n}, \underline{v}_{\mathrm{s}}^{n+\frac{1}{2}}\right)_{\phi^{n}, \Gamma_{N \text { nos }}^{n+1}}-\frac{\gamma \mu}{h}\left(\underline{\tilde{v}}_{\mathrm{f}}^{n+1}-\underline{v}_{\mathrm{s}}^{n-\frac{1}{2}}, \underline{v}_{\mathrm{s}}^{n+\frac{1}{2}}\right)_{\phi^{n}, \Gamma_{N \text { nos }}^{n+1}} .
\end{aligned}
$$


Considering now

$$
\begin{aligned}
& \mathcal{T}_{3}=\frac{\gamma \mu}{h}\left[\left(\underline{v}_{\mathrm{s}}^{n+\frac{1}{2}}-\underline{\tilde{v}}_{\mathrm{f}}^{n+1}, \underline{v}_{\mathrm{s}}^{n+\frac{1}{2}}\right)_{\phi^{n}, \Gamma_{N \text { nos }}^{n+1}}+\left(\underline{\tilde{v}}_{\mathrm{f}}^{n+1}-\underline{v}_{\mathrm{s}}^{n-\frac{1}{2}}, \underline{\tilde{v}}_{\mathrm{f}}^{n+1}\right)_{\phi^{n}, \Gamma_{N \text { nos }}^{n+1}}\right] \\
& =\frac{\gamma \mu}{h}\left[\left(\underline{v}_{\mathrm{s}}^{n+\frac{1}{2}}-\underline{\tilde{v}}_{\mathrm{f}}^{n+1}, \underline{v}_{\mathrm{s}}^{n+\frac{1}{2}}-\underline{\tilde{v}}_{\mathrm{f}}^{n+1}\right)_{\phi^{n}, \Gamma_{N \text { nos }}^{n+1}}+\left(\underline{v}_{\mathrm{s}}^{n+\frac{1}{2}}-\underline{\tilde{v}}_{\mathrm{f}}^{n+1}, \underline{\tilde{v}}_{\mathrm{f}}^{n+1}\right)_{\phi^{n}, \Gamma_{N \text { nos }}^{n+1}}\right. \\
& \left.+\left(\underline{\tilde{v}}_{\mathrm{f}}^{n+1}-\underline{v}_{\mathrm{s}}^{n-\frac{1}{2}}, \underline{\tilde{v}}_{\mathrm{f}}^{n+1}\right)_{\phi^{n}, \Gamma_{N \text { nos }}^{n+1}}\right] \\
& =\frac{\gamma \mu}{h}\left\|\underline{\tilde{v}}_{\mathrm{f}}^{n+1}-\underline{v}_{\mathrm{s}}^{n+\frac{1}{2}}\right\|_{\phi^{n}, \Gamma_{N \text { nos }}^{n+1}}^{2}+\frac{\gamma \mu}{h}\left(\underline{v}_{\mathrm{s}}^{n+\frac{1}{2}}-\underline{v}_{\mathrm{s}}^{n-\frac{1}{2}}, \underline{\tilde{v}}_{\mathrm{f}}^{n+1}\right)_{\phi^{n}, \Gamma_{N \text { nos }}^{n+1}},
\end{aligned}
$$

we have that

$\left(\underline{v}_{\mathrm{s}}^{n+\frac{1}{2}}-\underline{v}_{\mathrm{s}}^{n-\frac{1}{2}}, \underline{\tilde{v}}_{\mathrm{f}}^{n+1}\right)_{\phi^{n}, \Gamma_{N \mathrm{nos}}^{n+1}}=\left(\underline{v}_{\mathrm{s}}^{n+\frac{1}{2}}-\underline{v}_{\mathrm{s}}^{n-\frac{1}{2}}, \underline{\tilde{v}}_{\mathrm{f}}^{n+1}-\underline{v}_{\mathrm{s}}^{n+\frac{1}{2}}\right)_{\phi^{n}, \Gamma_{N \mathrm{nos}}^{n+1}}+\left(\underline{v}_{\mathrm{s}}^{n+\frac{1}{2}}-\underline{v}_{\mathrm{s}}^{n-\frac{1}{2}}, \underline{v}_{\mathrm{s}}^{n+\frac{1}{2}}\right)_{\phi^{n}, \Gamma_{N \text { nos }}^{n+1}}$.

Using $2(a, b) \leq\|a\|^{2}+\|b\|^{2}$ with the first term, and $2(a-b, a)=\|a\|^{2}-\|b\|^{2}+\|a-b\|^{2}$ with the second one, we obtain

$$
\begin{aligned}
\left(\underline{v}_{\mathrm{s}}^{n+\frac{1}{2}}-\underline{v}_{\mathrm{s}}^{n-\frac{1}{2}}, \underline{v}_{\mathrm{f}}^{n+1}\right)_{\phi^{n}, \Gamma_{N \mathrm{nos}}^{n+1}} & \geq-\frac{1}{2}\left\|\underline{v}_{\mathrm{s}}^{n+\frac{1}{2}}-\underline{v}_{\mathrm{s}}^{n-\frac{1}{2}}\right\|_{\phi^{n}, \Gamma_{N \mathrm{nos}}^{n+1}}^{2}-\frac{1}{2}\left\|\underline{\tilde{v}}_{\mathrm{f}}^{n+1}-\underline{v}_{\mathrm{s}}^{n+\frac{1}{2}}\right\|_{\phi^{n}, \Gamma_{N \mathrm{nos}}^{n+1}}^{2} \\
+ & \frac{1}{2}\left\|\underline{v}_{\mathrm{s}}^{n+\frac{1}{2}}-\underline{v}_{\mathrm{s}}^{n-\frac{1}{2}}\right\|_{\phi^{n}, \Gamma_{N \text { nos }}^{n+1}}^{2}+\frac{1}{2}\left\|\underline{v}_{\mathrm{s}}^{n+\frac{1}{2}}\right\|_{\phi^{n}, \Gamma_{N \mathrm{nos}}^{n+1}}^{2}-\frac{1}{2}\left\|\underline{v}_{\mathrm{s}}^{n-\frac{1}{2}}\right\|_{\phi^{n}, \Gamma_{N \mathrm{nos}}^{n+1}}^{2},
\end{aligned}
$$

hence,

$$
\mathcal{T}_{3} \geq \frac{\gamma \mu}{2 h}\left\|\underline{\tilde{v}}_{\mathrm{f}}^{n+1}-\underline{v}_{\mathrm{s}}^{n+\frac{1}{2}}\right\|_{\phi^{n}, \Gamma_{N \text { nos }}^{n+1}}^{2}+\frac{\gamma \mu}{2 h}\left\|\underline{\mathrm{s}}_{\mathrm{s}}^{n+\frac{1}{2}}\right\|_{\phi^{n}, \Gamma_{N \text { nos }}^{n+1}}^{2}-\frac{\gamma \mu}{2 h}\left\|\underline{v}_{\mathrm{s}}^{n-\frac{1}{2}}\right\|_{\phi^{n}, \Gamma_{N \text { nos }}^{n+1}}^{2} .
$$

For the term $\mathcal{T}_{2}$, we use Young's inequality $(a, b) \leq \frac{1}{2 L}\|a\|^{2}+\frac{L}{2}\|b\|^{2}$, with $L$ homogeneous to a length here, to get

$$
-\mathcal{T}_{2} \leq \mu\left[L\left\|\underline{\underline{\varepsilon}}\left(\underline{\tilde{v}}_{\mathrm{f}}^{n+1}\right) \cdot \underline{n}\right\|_{\phi^{n}, \Gamma_{N \mathrm{nos}}^{n+1}}^{2}+\frac{1}{L}\left\|\underline{\tilde{v}}_{\mathrm{f}}^{n+1}-\underline{v}_{\mathrm{s}}^{n+\frac{1}{2}}\right\|_{\phi^{n}, \Gamma_{N \mathrm{nos}}^{n+1}}^{2}\right],
$$

and the inverse inequality (11) then gives

$$
\mathcal{T}_{2} \geq-\mu \frac{C_{i e} L}{h}\left\|\underline{\underline{\varepsilon}}\left(\underline{\tilde{v}}_{\mathrm{f}}^{n+1}\right)\right\|_{\phi^{n}, \Omega_{\mathrm{f}}^{n+1}}^{2}-\frac{\mu}{L}\left\|\underline{\tilde{v}}_{\mathrm{f}}^{n+1}-\underline{v}_{\mathrm{s}}^{n+\frac{1}{2}}\right\|_{\phi^{n}, \Gamma_{N \text { nos }}^{n+1}}^{2} .
$$

Using the bounds (19) and (20) in (18), we obtain

$$
\begin{aligned}
& \frac{\mathcal{K}_{\mathrm{f}}^{n+1}-\mathcal{K}_{\mathrm{f}}^{n}}{\Delta t}+\frac{\mathcal{K}_{\mathrm{s}}^{n+1}-\mathcal{K}_{\mathrm{s}}^{n}}{\Delta t}+\int_{\Omega^{0}}\left(\left.\frac{\partial \Psi}{\partial \underline{\underline{e}}}\right|^{n+\frac{1}{2} \sharp}+\left.\frac{\partial \Psi^{\mathrm{damp}}}{\partial \underline{\underline{e}}}\right|^{n+\frac{1}{2} \sharp}\right): d_{\underline{y}} \underline{\underline{e}}^{n+\frac{1}{2} \sharp} \cdot \underline{v}_{\mathrm{s}}^{n+\frac{1}{2}} d \Omega \\
& -\underbrace{}_{\Omega_{\mathrm{f}}^{n+1}} p^{n+1}\left(\underline{\nabla}_{\underline{\mathrm{x}}} \phi^{n}\right) \cdot\left(\underline{v}_{\mathrm{f}}^{n+1}-\underline{v}_{\mathrm{s}}^{n+\frac{1}{2}}\right) d \Omega-\int_{\Omega_{\mathrm{f}}^{n+1}} p^{n+1} \phi^{n} \underline{\nabla}_{\underline{\mathrm{x}}} \cdot\left(\underline{v}_{\mathrm{f}}^{n+1}-\underline{v}_{\mathrm{s}}^{n+\frac{1}{2}}\right) d \Omega \\
& +\frac{\rho_{\mathrm{f}}}{2 \Delta t}\left\|\underline{\tilde{v}}_{\mathrm{f}}^{n+1}-\underline{v}_{\mathrm{f}}^{n}\right\|_{\phi^{n-1}, \Omega_{\mathrm{f}}^{n}}^{2}+\frac{\rho_{\mathrm{f}}}{2 \Delta t}\left\|\underline{v}_{\mathrm{f}}^{n+1}-\underline{\tilde{v}}_{\mathrm{f}}^{n+1}\right\|_{\phi^{n}, \Omega_{\mathrm{f}}^{n+1}}^{2}+\frac{\gamma \mu}{2 h}\left\|\underline{v}_{\mathrm{s}}^{n+\frac{1}{2}}\right\|_{\phi^{n}, \Gamma_{N \text { nos }}^{n+1}}^{2}-\frac{\gamma \mu}{2 h}\left\|\underline{v}_{\mathrm{s}}^{n-\frac{1}{2}}\right\|_{\phi^{n}, \Gamma_{N \text { nos }}^{n+1}}^{2} \\
& +\mu\left(2-\frac{C_{i e} L}{h}\right)\left\|\underline{\underline{\varepsilon}}\left(\underline{\tilde{v}}_{\mathrm{f}}^{n+1}\right)\right\|_{\phi^{n}, \Omega_{\mathrm{f}}^{n+1}}^{2}+\mu\left(\frac{\gamma}{2 h}-\frac{1}{L}\right)\left\|\underline{\tilde{v}}_{\mathrm{f}}^{n+1}-\underline{v}_{\mathrm{s}}^{n+\frac{1}{2}}\right\|_{\phi^{n}, \Gamma_{N \mathrm{nos}}^{n+1}}^{2} \\
& \leq\left(\underline{t}^{n+1}, \underline{v}_{\mathrm{f}}^{n+1}\right)_{\phi^{n}, \Gamma_{N}^{n+1}}+\left(\underline{t}_{0}^{n+1}, \underline{v}_{\mathrm{s}}^{n+\frac{1}{2}}\right)_{1-\phi^{n}, \Gamma_{N}^{0}-\mathcal{T}_{1}} \\
& -\int_{\Omega_{\mathrm{f}}^{n+1}}\left(\underline{v}_{\mathrm{f}}^{n+1}-\underline{v}_{\mathrm{s}}^{n+\frac{1}{2}}\right) \cdot\left|\phi^{n}\right|^{2} \underline{\underline{D}}_{\mathrm{f}} \cdot\left(\underline{v}_{\mathrm{f}}^{n+1}-\underline{v}_{\mathrm{s}}^{n+\frac{1}{2}}\right) d \Omega \text {. }
\end{aligned}
$$


Now, using $\underline{\nabla} \cdot\left(\phi \underline{v}_{\mathrm{f}}\right)=\underline{\nabla} \phi \cdot \underline{v}_{\mathrm{f}}+\phi \underline{\nabla} \cdot \underline{v}_{\mathrm{f}}$, and $(7 \mathrm{a})$ evaluated with $\frac{p^{n+1}}{\rho_{\mathrm{f}}}-$ again, assuming this is allowed by the spatial discretization, see Section 3.3 - we have

$$
\begin{aligned}
\mathcal{T}_{4} & =-\int_{\Omega_{\mathrm{f}}^{n+1}} p^{n+1}\left(\underline{\nabla}_{\underline{\mathrm{x}}} \phi^{n}\right) \cdot\left(\underline{v}_{\mathrm{f}}^{n+1}-\underline{v}_{\mathrm{s}}^{n+\frac{1}{2}}\right) d \Omega-\int_{\Omega_{\mathrm{f}}^{n+1}} p^{n+1} \phi^{n} \underline{\nabla}_{\underline{\mathrm{x}}} \cdot\left(\underline{v}_{\mathrm{f}}^{n+1}-\underline{v}_{\mathrm{s}}^{n+\frac{1}{2}}\right) d \Omega \\
& =-\int_{\Omega_{\mathrm{f}}^{n+1}} p^{n+1} \underline{\nabla}_{\underline{\mathrm{x}}} \cdot\left(\phi^{n}\left(\underline{v}_{\mathrm{f}}^{n+1}-\underline{v}_{\mathrm{s}}^{n+\frac{1}{2}}\right)\right) d \Omega \\
& =\int_{\Omega^{0}} \frac{p^{n+1}}{\rho_{\mathrm{f}}} \frac{m^{n+1}-m^{n}}{\Delta t} d \Omega \\
& =\left.\int_{\Omega^{0}} \frac{\partial \Psi}{\partial m}\right|^{n+\frac{1}{2} \sharp} \frac{m^{n+1}-m^{n}}{\Delta t} d \Omega .
\end{aligned}
$$

Furthermore, thanks to (9) we have

$$
\begin{aligned}
\left.\frac{\partial \Psi}{\partial \underline{\underline{e}}}\right|^{n+\frac{1}{2} \sharp}: d_{\underline{y}} \underline{\underline{e}} \underline{\underline{e}}^{n+\frac{1}{2} \sharp} \cdot \underline{v}_{\mathrm{s}}^{n+\frac{1}{2}} & +\left.\frac{\partial \Psi}{\partial m}\right|^{n+\frac{1}{2} \sharp} \frac{m^{n+1}-m^{n}}{\Delta t} \\
& =\left.\frac{\partial \Psi}{\partial \underline{\underline{e}}}\right|^{n+\frac{1}{2} \sharp}: \frac{\underline{\underline{e}}^{n+1}-\underline{\underline{e}}^{n}}{\Delta t}+\left.\frac{\partial \Psi}{\partial m}\right|^{n+\frac{1}{2} \sharp} \frac{m^{n+1}-m^{n}}{\Delta t} \\
& =\frac{\Psi^{\mathrm{n}+1}-\Psi^{\mathrm{n}}}{\Delta t} .
\end{aligned}
$$

Finally, we are led to

$$
\begin{gathered}
\frac{\mathcal{E}^{n+1}-\mathcal{E}^{n}}{\Delta t}+\frac{\gamma \mu}{2 h}\left\|\underline{v}_{\mathrm{s}}^{n+\frac{1}{2}}\right\|_{\phi^{n}, \Gamma_{N \text { nos }}^{n+1}}^{2}-\frac{\gamma \mu}{2 h}\left\|\underline{v}_{\mathrm{s}}^{n-\frac{1}{2}}\right\|_{\phi^{n}, \Gamma_{N \text { nos }}^{n+1}}^{2} \\
\leq\left(\underline{t}^{n+1}, \underline{v}_{\mathrm{f}}^{n+1}\right)_{\phi^{n}, \Gamma_{N}^{n+1}}+\left(\underline{t}_{0}^{n+1}, \underline{v}_{\mathrm{s}}^{n+\frac{1}{2}}\right)_{1-\phi^{n}, \Gamma_{N}^{0}}-\left.\int_{\Omega^{0}} \frac{\partial \Psi^{\mathrm{damp}}}{\partial \underline{\dot{e}}}\right|^{n+\frac{1}{2} \sharp}: d_{\underline{y}} \underline{\underline{e}}^{n+\frac{1}{2} \sharp} \cdot \underline{v}_{\mathrm{s}}^{n+\frac{1}{2}} d \Omega \\
\quad-\int_{\Omega_{\mathrm{f}}^{n+1}}\left(\underline{v}_{\mathrm{f}}^{n+1}-\underline{v}_{\mathrm{s}}^{n+\frac{1}{2}}\right) \cdot\left|\phi^{n}\right|^{2} \underline{D}_{\mathrm{f}} \cdot\left(\underline{v}_{\mathrm{f}}^{n+1}-\underline{v}_{\mathrm{s}}^{n+\frac{1}{2}}\right) d \Omega-\mathcal{T}_{1} \\
-\frac{\rho_{\mathrm{f}}}{2 \Delta t}\left\|\underline{\tilde{v}}_{\mathrm{f}}^{n+1}-\underline{v}_{\mathrm{f}}^{n}\right\|_{\phi^{n-1}, \Omega_{\mathrm{f}}^{n}}^{2}-\frac{\rho_{\mathrm{f}}}{2 \Delta t}\left\|\underline{\mathrm{f}}_{\mathrm{f}}^{n+1}-\underline{\tilde{v}}_{\mathrm{f}}^{n+1}\right\|_{\phi^{n}, \Omega_{\mathrm{f}}^{n+1}}^{2} \\
\left.-\mu\left(2-\frac{C_{i e} L}{h}\right) \| \underline{\varepsilon}_{\tilde{v}_{\mathrm{f}}^{n+1}}\right)\left\|_{\phi^{n}, \Omega_{\mathrm{f}}^{n+1}}^{2 n}-\mu\left(\frac{\gamma}{2 h}-\frac{1}{L}\right)\right\| \underline{\tilde{v}}_{\mathrm{f}}^{n+1}-\underline{v}_{\mathrm{s}}^{n+\frac{1}{2}} \|_{\phi^{n}, \Gamma_{N \text { nos }}^{n+1}}^{2}
\end{gathered}
$$

Therefore, we can have (12) provided we can find $L$ such that $2-\frac{C_{i e} L}{h} \geq 0$ and $\frac{\gamma}{2 h}-\frac{1}{L}>0$, i.e. when $\gamma>C_{i e}$.

\section{Remark 4}

Proposition 2 ensures the energy stability of our scheme provided $\gamma \mu \Delta t=O(h)$. This CFL-like condition and the interface term $\frac{\gamma \mu}{2 h}\left\|\underline{v}_{\mathrm{s}}^{n+\frac{1}{2}}\right\|_{\phi^{n}, \Gamma_{N \text { nos }}}^{2}$, that could be incorporated to the numerical energy if $\Gamma_{N \text { nos }}$ was fixed, already appeared in the Robin based fluid-structure interaction splitting scheme of $[1]$.

\section{Remark 5}

All the physical dissipation terms already seen in the continuous energy balance (5) are present in this discrete balance. In addition, as in [1], the terms $\left\|\underline{\tilde{v}}_{\mathrm{f}}^{n+1}-\underline{v}_{\mathrm{f}}^{n}\right\|_{\phi^{n-1}, \Omega_{\mathrm{f}}^{n}}^{2},\left\|\underline{\mathrm{v}}_{\mathrm{f}}^{n+1}-\underline{\tilde{v}}_{\mathrm{f}}^{n+1}\right\|_{\phi^{n}, \Omega_{\mathrm{f}}^{n+1}}^{2}$ and $\left\|\underline{\tilde{v}}_{\mathrm{f}}^{n+1}-\underline{v}_{\mathrm{s}}^{n+\frac{1}{2}}\right\|_{\phi^{n}, \Gamma_{N \text { nos }}^{n+1}}^{2}$ bring numerical dissipation. 


\section{Remark 6}

In line with Remark 1, let us mention that this stability analysis can easily be reduced to a fluidstructure interaction problem in the ALE formalism. Nevertheless, one noticeable difference is that (16) - a crucial ingredient of the stability analysis that rests here on (7a) and the definition of $\phi$ - would then require a "geometric conservation law" (GCL), see e.g. Proposition 4.7.1 in [37], or (9.53) in [20]. Indeed, this type of condition is classically used to ensure stability at the discrete level with moving domains $[16,17,20,24,38,40]$.

\subsection{Consistency considerations pertaining to spatial discretization}

In this section, we provide some insight on consistency perturbations that may arise in the above energy estimate due to spatial discretization. First of all, since the porosity is computed explicitly at Step 0 of our algorithm, it is natural to compute it exactly as $\phi^{n}=\frac{m^{n} / \rho_{\mathrm{f}}+\phi_{0}}{J\left(y_{\mathrm{s}}^{n}\right)}$ at the quadrature points, and store these values for use in the next steps. Then, in order for (21) to hold, we recall that the discrete spaces for the pressure and the mass need to coincide, a reasonable choice that we will make. However, in this case we need to adapt the pressure law at Step 2a into

$$
p^{n+1} \hat{=} \pi_{m}\left(\left.\rho_{\mathrm{f}} \frac{\partial \Psi}{\partial m}\right|^{n+\frac{1}{2} \sharp}\right),
$$

where $\pi_{m}$ denotes a projection operator onto the discrete space chosen for the fluid mass. In our case we will consider $L^{2}$-projection. Then, in our above stability analysis, the only modification induced pertains to (16), for which we now have by testing (7a) at time step $n-1$ with $q_{h}^{*}=\pi_{m}\left(\left|\underline{\underline{f}}_{\mathrm{f}}^{n+1}\right|^{2}\right)$

$$
\begin{aligned}
\frac{1}{2} \int_{\Omega_{\mathrm{f}}^{n}}\left|\underline{\tilde{v}}_{\mathrm{f}}^{n+1}\right|^{2} \underline{\nabla}_{\underline{\mathrm{x}}} \cdot\left(\rho_{\mathrm{f}} \phi^{n-1}\left(\underline{v}_{\mathrm{f}}^{n}-\underline{v}_{\mathrm{s}}^{n-\frac{1}{2}}\right)\right) d \Omega \\
=\frac{1}{2} \int_{\Omega_{\mathrm{f}}^{n}} \pi_{m}\left(\left|\underline{\tilde{v}}_{\mathrm{f}}^{n+1}\right|^{2}\right) \underline{\nabla}_{\underline{\mathrm{x}}} \cdot\left(\rho_{\mathrm{f}} \phi^{n-1}\left(\underline{v}_{\mathrm{f}}^{n}-\underline{v}_{\mathrm{s}}^{n-\frac{1}{2}}\right)\right) d \Omega+\mathcal{S}_{1} \\
=-\frac{1}{2} \int_{\Omega^{0}} \frac{m^{n}-m^{n-1}}{\Delta t} \pi_{m}\left(\left|\underline{\tilde{v}}_{\mathrm{f}}^{n+1}\right|^{2}\right) d \Omega+\mathcal{S}_{1} \\
=-\frac{1}{2} \int_{\Omega^{0}} \frac{\phi^{n} \rho_{\mathrm{f}} J\left(\underline{y}_{\mathrm{s}}^{n}\right)-\phi^{n-1} \rho_{\mathrm{f}} J\left(\underline{y}_{\mathrm{s}}^{n-1}\right)}{\Delta t} \pi_{m}\left(\left|\underline{\underline{v}}_{\mathrm{f}}^{n+1}\right|^{2}\right) d \Omega+\mathcal{S}_{1} \\
=-\frac{\rho_{\mathrm{f}}}{2 \Delta t}\left(\left\|\left(\pi_{m}\left(\left|\underline{\tilde{v}}_{\mathrm{f}}^{n+1}\right|^{2}\right)\right)^{\frac{1}{2}}\right\|_{\phi^{n}, \Omega_{\mathrm{f}}^{n+1}}^{2}-\left\|\left(\pi_{m}\left(\left|\underline{v}_{\mathrm{f}}^{n+1}\right|^{2}\right)\right)^{\frac{1}{2}}\right\|_{\phi^{n-1}, \Omega_{\mathrm{f}}^{n}}^{2}\right)+\mathcal{S}_{1} \\
=-\frac{\rho_{\mathrm{f}}}{2 \Delta t}\left(\left\|\underline{v}_{\mathrm{f}}^{n+1}\right\|_{\phi^{n}, \Omega_{\mathrm{f}}^{n+1}}^{2}-\left\|\underline{\tilde{v}}_{\mathrm{f}}^{n+1}\right\|_{\phi^{n-1}, \Omega_{\mathrm{f}}^{n}}^{2}\right)+\mathcal{S}_{1}+\mathcal{S}_{2},
\end{aligned}
$$

with the following consistency perturbations induced in (16), i.e. our specific version of the GCL,

$$
\left\{\begin{array}{c}
\mathcal{S}_{1}=\frac{1}{2} \int_{\Omega_{\mathrm{f}}^{n}}\left(\left|\underline{\tilde{v}}_{\mathrm{f}}^{n+1}\right|^{2}-\pi_{m}\left(\left|\underline{\tilde{v}}_{\mathrm{f}}^{n+1}\right|^{2}\right)\right) \underline{\nabla}_{\underline{\mathrm{x}}} \cdot\left(\rho_{\mathrm{f}} \phi^{n-1}\left(\underline{v}_{\mathrm{f}}^{n}-\underline{v}_{\mathrm{s}}^{n-\frac{1}{2}}\right)\right) d \Omega \\
\mathcal{S}_{2}=\frac{\rho_{\mathrm{f}}}{2 \Delta t}\left(\left\|\underline{\tilde{v}}_{\mathrm{f}}^{n+1}\right\|_{\phi^{n}, \Omega_{\mathrm{f}}^{n+1}}^{2}-\left\|\underline{\tilde{v}}_{\mathrm{f}}^{n+1}\right\|_{\phi^{n-1}, \Omega_{\mathrm{f}}^{n}}^{2}\right. \\
\left.\quad-\left\|\left(\pi_{m}\left(\left|\underline{\tilde{v}}_{\mathrm{f}}^{n+1}\right|^{2}\right)\right)^{\frac{1}{2}}\right\|_{\phi^{n}, \Omega_{\mathrm{f}}^{n+1}}^{2}+\left\|\left(\pi_{m}\left(\left|\underline{\tilde{v}}_{\mathrm{f}}^{n+1}\right|^{2}\right)\right)^{\frac{1}{2}}\right\|_{\phi^{n-1}, \Omega_{\mathrm{f}}^{n}}^{2}\right)
\end{array}\right.
$$


Therefore, the final energy balance (22) is modified into

$$
\begin{aligned}
& \frac{\mathcal{E}^{n+1}-\mathcal{E}^{n}}{\Delta t}+\frac{\gamma \mu}{2 h}\left\|\underline{v}_{\mathrm{s}}^{n+\frac{1}{2}}\right\|_{\phi^{n}, \Gamma_{N \text { nos }}^{n+1}}^{2}-\frac{\gamma \mu}{2 h}\left\|\underline{v}_{\mathrm{s}}^{n-\frac{1}{2}}\right\|_{\phi^{n}, \Gamma_{N \text { nos }}^{n+1}}^{2} \\
& \leq\left(\underline{t}^{n+1}, \underline{v}_{\mathrm{f}}^{n+1}\right)_{\phi^{n}, \Gamma_{N}^{n+1}}+\left(\underline{t}_{0}^{n+1}, \underline{v}_{\mathrm{s}}^{n+\frac{1}{2}}\right)_{1-\phi^{n}, \Gamma_{N}^{0}}-\left.\int_{\Omega^{0}} \frac{\partial \Psi^{\mathrm{damp}}}{\partial \underline{\dot{e}}}\right|^{n+\frac{1}{2} \sharp}: d_{\underline{y}} \underline{\underline{e}}^{n+\frac{1}{2} \sharp} \cdot \underline{v}_{\mathrm{s}}^{n+\frac{1}{2}} d \Omega \\
& -\int_{\Omega_{\mathrm{f}}^{n+1}}\left(\underline{v}_{\mathrm{f}}^{n+1}-\underline{v}_{\mathrm{s}}^{n+\frac{1}{2}}\right) \cdot\left|\phi^{n}\right|^{2} \underline{\underline{D}}_{\mathrm{f}} \cdot\left(\underline{v}_{\mathrm{f}}^{n+1}-\underline{v}_{\mathrm{s}}^{n+\frac{1}{2}}\right) d \Omega-\mathcal{T}_{1}-\mathcal{S}_{1}-\mathcal{S}_{2} \\
& +\frac{\rho_{\mathrm{f}}}{2 \Delta t}\left\|\underline{\tilde{v}}_{\mathrm{f}}^{n+1}-\underline{v}_{\mathrm{f}}^{n}\right\|_{\phi^{n-1}, \Omega_{\mathrm{f}}^{n}}^{2}+\frac{\rho_{\mathrm{f}}}{2 \Delta t}\left\|\underline{v}_{\mathrm{f}}^{n+1}-\underline{\tilde{\tau}}_{\mathrm{f}}^{n+1}\right\|_{\phi^{n}, \Omega_{\mathrm{f}}^{n+1}}^{2} \\
& -\mu\left(2-\frac{C_{i e} L}{h}\right)\left\|\underline{\underline{\varepsilon}}\left(\underline{\tilde{v}}_{\mathrm{f}}^{n+1}\right)\right\|_{\phi^{n}, \Omega_{\mathrm{f}}^{n+1}}^{2}-\mu\left(\frac{\gamma}{2 h}-\frac{1}{L}\right)\left\|\underline{\tilde{v}}_{\mathrm{f}}^{n+1}-\underline{v}_{\mathrm{s}}^{n+\frac{1}{2}}\right\|_{\phi^{n}, \Gamma_{N \mathrm{nos}}^{n+1}}^{2} .
\end{aligned}
$$

\section{Remark 7 (Pressure discretization)}

When the free energy $\Psi$ is quadratic in $m$ - a rather common assumption - the pressure and fluid mass are linearly related by the pressure law, and therefore assuming that the corresponding discrete spaces are identical we can drop the projection in (23). In addition, it should be pointed out that specific numerical issues - namely, numerical locking and pressure instabilities - are likely to arise when the solid constituent is considered as nearly incompressible - i.e. with a large bulk modulus associated with $J_{\mathrm{s}}$ in $\Psi_{\mathrm{s}}\left(\underline{\underline{e}}, J_{\mathrm{s}}\right)$ - due to the similarity of $(7)$ with a Stokes problem, but this topic lies beyond the scope of the present article.

\section{$4 \quad$ Numerical illustrations}

In this section, we present various numerical results to illustrate the behavior of the poroelastic model under large deformations, simulated with our proposed method, namely, two test problems inspired from [9] - see also [41] - and the detailed numerical monitoring of the energy balance considered in the above stability analysis. The free energy considered in these examples is given by

$$
\Psi=\Psi^{\text {skel }}+\Psi^{\text {bulk }}+\Psi^{\text {por }},
$$

where $\Psi^{\text {skel }}$ is potential of Ciarlet-Geymonat type, i.e.

$$
\Psi^{\mathrm{skel}}=\kappa_{1}\left(I_{1} I_{3}^{-\frac{1}{3}}-3\right)+\kappa_{2}\left(I_{2} I_{3}^{-\frac{2}{3}}-3\right)+\kappa\left(\sqrt{I_{3}}-1-\ln \left(\sqrt{I_{3}}\right)\right)
$$

with the classical invariants

$$
I_{1}=\operatorname{tr}(\underline{\underline{C}}), \quad I_{2}=\frac{1}{2}\left((\operatorname{tr} \underline{\underline{C}})^{2}-\operatorname{tr}\left(\underline{\underline{C}}^{2}\right)\right), \quad I_{3}=\operatorname{det}(\underline{\underline{C}}) .
$$

The term

$$
\Psi^{\text {bulk }}\left(J_{\mathrm{s}}\right)=\kappa_{\mathrm{s}}\left(\frac{J_{\mathrm{s}}}{1-\phi_{0}}-1-\ln \left(\frac{J_{\mathrm{s}}}{1-\phi_{0}}\right)\right)
$$

governs the compressibility of the solid constituent, and the last term

$$
\Psi^{\text {por }}=-\eta_{\text {por }} \ln \left(\frac{m}{\rho_{\mathrm{f}}}+\phi_{0}\right)
$$

aims at preventing the porosity $\phi$ from taking negative values, see [10]. The parameter values common to the three test problems are $\kappa=\kappa_{1}=210^{3}, \kappa_{2}=33, \rho_{\mathrm{s}}=\rho_{\mathrm{f}}=10^{3}, \mu=0.035$, $\phi_{0}=0.1$ and $\gamma=20$ (all SI), while Table 1 gives the parameter values that differ. The same 2D 


\begin{tabular}{l||l|l|l|l|l|l|} 
Parameter & $\left|\Omega^{0}\right|$ & $\Delta t$ & $\kappa_{\mathrm{s}}$ & $\eta_{\mathrm{d}}$ & $\eta_{\mathrm{por}}$ & $\underline{\underline{\underline{D}}}_{\mathrm{f}}$ \\
\hline Swelling & $10^{-4}$ & $10^{-3}$ & $210^{3}$ & 0 & 0 & $10^{7} \underline{\underline{\underline{I}}}$ \\
Drainage & $10^{-6}$ & $210^{-6}$ & $210^{5}$ & 68 & 1.5 & $410^{5} \underline{\underline{I}}$ \\
Energy & $10^{-4}$ & $10^{-4}$ & $210^{3}$ & 0 & 0 & $10^{7} \underline{\underline{\underline{I}}}$
\end{tabular}

Table 1: Parameters with different values among test problems (SI units).

square geometry is considered in all test problems, albeit with different dimensions as specified in the table. The mesh is obtained by a regular splitting of the domain into 72 triangular elements, as shown in Fig. 1. As discretization spaces we use continuous- $P 1$ elements for the solid displacement and for the fluid mass, as well as for the pressure. For the fluid velocities, due to the Stokes-like nature of Step 2 a we use continuous- $P 1$ with an additional internal "bubble" degree of freedom.

\section{Remark 8 (Calibration of stabilization constant)}

Regarding the choice of the stabilization constant $\gamma$, the mathematical analysis of Proposition 2 has shown that it should be conditioned by the lower bound given by $C_{i e}$, the constant in the inverse inequality (11). For the latter constant, a rough estimate obtained by assuming linear shape functions and constant fluid fraction would be 2 in 2D (3 in 3D). In practice, we have chosen $\gamma=20$ to be conservative - see also Remark 3 - and after numerically checking that in this case the impact of the penalization term in the overall energy balance remains small.

\subsection{Implementation considerations}

We implemented our proposed method in FreeFem $++[26]$, for the two-dimensional version of the poromechanical model.

Three Newton algorithms are implemented in the code, namely,

1. for the solution of the fluid-pressure problem (7);

2. for the solution of the solid problem (8);

3. for coupling these two sub-steps within the implicit step.

We used the same convergence criteria for these three iteration loops, i.e. tolerance parameters for the absolute and relative - with respect to initialization step - values of the residuals, set to $10^{-7}$ and $10^{-13}$, respectively.

The FreeFem++ environment is very powerful, in particular for prototyping purposes, but does not allow as much control as an in-house finite element software on the numerical operators to be implemented. For this reason, we resorted to several simplifications in this numerical study, namely:

- for $\left.\frac{\partial \Psi}{\partial \underline{\underline{e}}}\right|^{n+\frac{1}{2} \sharp}$ we used the simpler rule

$$
\left.\frac{\partial \Psi}{\partial \underline{\underline{e}}}\right|^{n+\frac{1}{2} \sharp} \hat{=} \frac{\partial \Psi}{\partial \underline{\underline{e}}}\left(\underline{\underline{e}}^{n+\frac{1}{2} \sharp}, m^{n+1}\right),
$$

as already considered and assessed in [25], instead of the approach of [22] considered in $(9 b)$; 
- in addition, the convective term in (6) - namely, the third term - was computed on the domain $\Omega^{n+1}$ instead of $\Omega^{n}$, which leads us to changing $\mathcal{S}_{1}$ into

$$
\begin{aligned}
\mathcal{S}_{1}^{\prime}=\frac{1}{2} \int_{\Omega_{\mathrm{f}}^{n+1}}\left|\underline{\tilde{v}}_{\mathrm{f}}^{n+1}\right|^{2} \underline{\nabla}_{\underline{\mathrm{x}}} \cdot\left(\rho_{\mathrm{f}} \phi^{n-1}\left(\underline{v}_{\mathrm{f}}^{n}-\underline{v}_{\mathrm{s}}^{n-\frac{1}{2}}\right)\right) d \Omega \\
\quad-\frac{1}{2} \int_{\Omega_{\mathrm{f}}^{n}} \pi_{m}\left(\left|\underline{\tilde{v}}_{\mathrm{f}}^{n+1}\right|^{2}\right) \underline{\nabla}_{\underline{\mathbf{x}}} \cdot\left(\rho_{\mathrm{f}} \phi^{n-1}\left(\underline{v}_{\mathrm{f}}^{n}-\underline{v}_{\mathrm{s}}^{n-\frac{1}{2}}\right)\right) d \Omega ;
\end{aligned}
$$

- finally, the computation of $\phi$ at Step 0 . is performed weakly by $L^{2}$ projection in the finite element space, instead of exactly at quadrature points as specified. This introduces an extra consistency perturbation to be added to $\mathcal{S}_{1}$ and $\mathcal{S}_{2}$, viz.

$$
\mathcal{S}_{3}=\frac{1}{2} \int_{\Omega^{0}}\left(\frac{\phi^{n} \rho_{\mathrm{f}} J\left(\underline{y}_{\mathrm{s}}^{n}\right)-\phi^{n-1} \rho_{\mathrm{f}} J\left(\underline{y}_{\mathrm{s}}^{n-1}\right)}{\Delta t}-\frac{m^{n}-m^{n-1}}{\Delta t}\right) \pi_{m}\left(\left|\underline{\tilde{v}}_{\mathrm{f}}^{n+1}\right|^{2}\right) d \Omega .
$$

Nevertheless, we provide below a numerical assessment of the energy balance in order to check that these simplifications do not significantly affect this balance. In what follows, we introduce the following notation

$$
\mathcal{S}_{4}=\frac{\gamma \mu}{2 h}\left\|\underline{v}_{\mathrm{s}}^{n+\frac{1}{2}}\right\|_{\phi^{n}, \Gamma_{N \mathrm{nos}}^{n+1}}^{2}-\frac{\gamma \mu}{2 h}\left\|\underline{\mathrm{s}}_{\mathrm{n}}^{n-\frac{1}{2}}\right\|_{\phi^{n}, \Gamma_{N \mathrm{nos}}^{n+1}}^{2} .
$$

\subsection{Swelling test under porous flow}

In the swelling test, no external force is applied to the system, and a gradual pressure increase is prescribed on the inlet side, see Fig. 1 , in the form $p_{\text {ext }}=10^{3}\left(1-\exp -t^{2} / 0.25\right)$ as a Neumann boundary condition applied on the fluid only, while maintaining $p_{\text {ext }}=0$ on the outlet side (on the right of the sample). The top and bottom sides are assumed to be of no-sliding type $\left(\underline{v}_{\mathrm{f}}=\underline{v}_{\mathrm{s}}\right)$, and normal displacements are prevented for the solid on the left and bottom sides. We have no volume-distributed fluid source nor sink. As expected, the system is gradually filled with fluid until a stationary state is reached, in which the fluid pressure is in equilibrium with elastic forces. The simulations results presented in Fig. 1 are consistent with those given in [9].

\subsection{Drainage test}

In the drainage test, geometry and solid essential boundary conditions are the same as in the swelling test. An external pressure $p_{\text {ext }}=10^{4}\left(1-\exp -t^{2} / 0.04\right)$ is applied on all the sides of the square. All sides are of no-sliding type, and a volume-distributed sink linearly related to the pressure $\left(\theta=-\rho_{\mathrm{f}} \beta\left(p-p_{\text {sink }}\right)\right.$, with $p_{\text {sink }}=0$ and $\left.\beta=0.01\right)$ allows the fluid to escape the material. As shown in Fig. 2, in a first phase, the fluid is drained out of the system nearly completely, until $m / \rho_{\mathrm{f}}$ approaches -0.1 (i.e. $\phi \approx 0$ ). Then, only the solid phase remains, and the material behaves like a standard solid, that compresses according to its bulk modulus $\kappa_{\mathrm{s}}$. Solutions are here homogeneous in space, and we can verify that the solutions satisfy the following identity that comes from (7a)

$$
\frac{m^{n+1}-m^{n}}{\rho_{\mathrm{f}} \Delta t}=-J^{n} \beta p^{n} .
$$

This illustrates how the penalization term $\Psi^{\text {por }}$ used in the energy is effective to prevent $\phi$ from reaching negative values. 
Skeleton
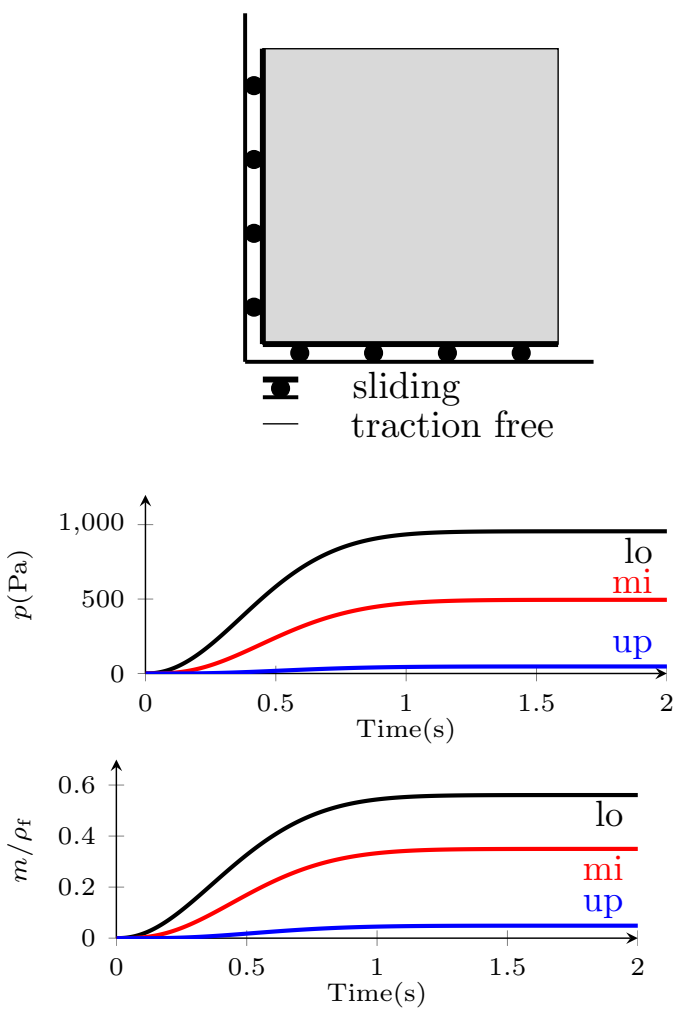

Fluid
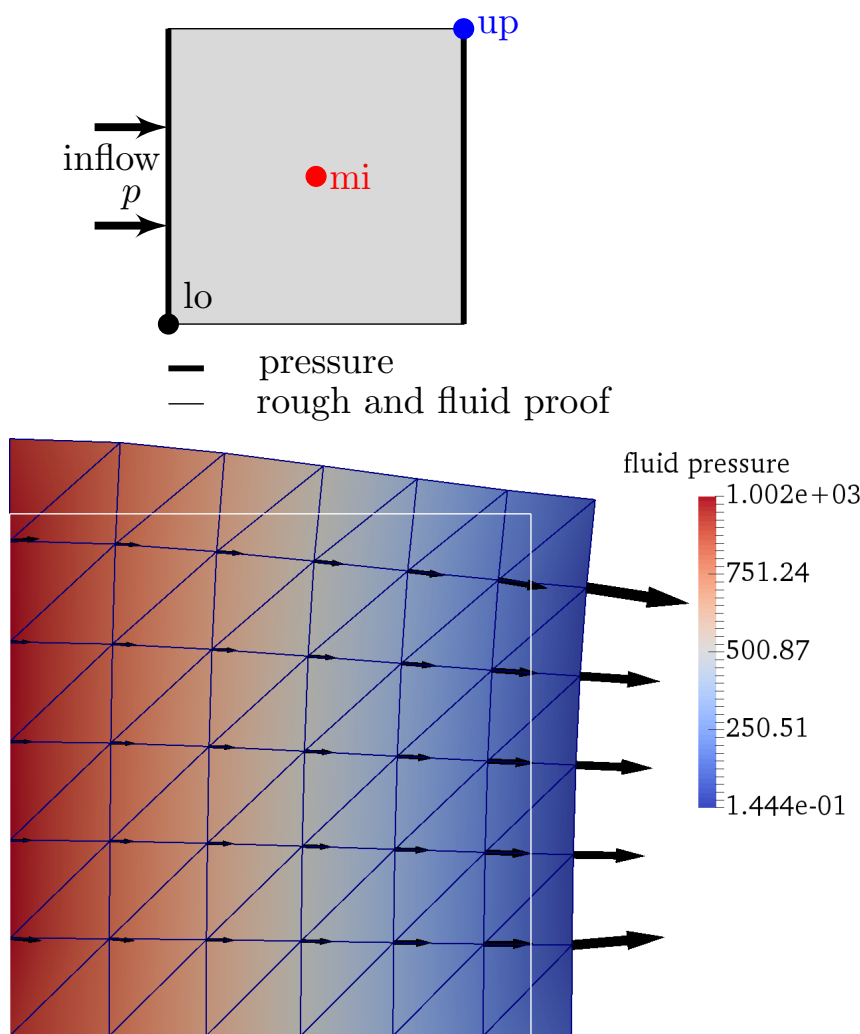

Figure 1: Swelling under porous flow. Top row: geometry and boundary conditions - Bottom row: (left) fluid pressure and mass at three points shown on top-right; (right) fluid velocity and pressure at steady state on deformed configuration, with initial configuration contour in white 

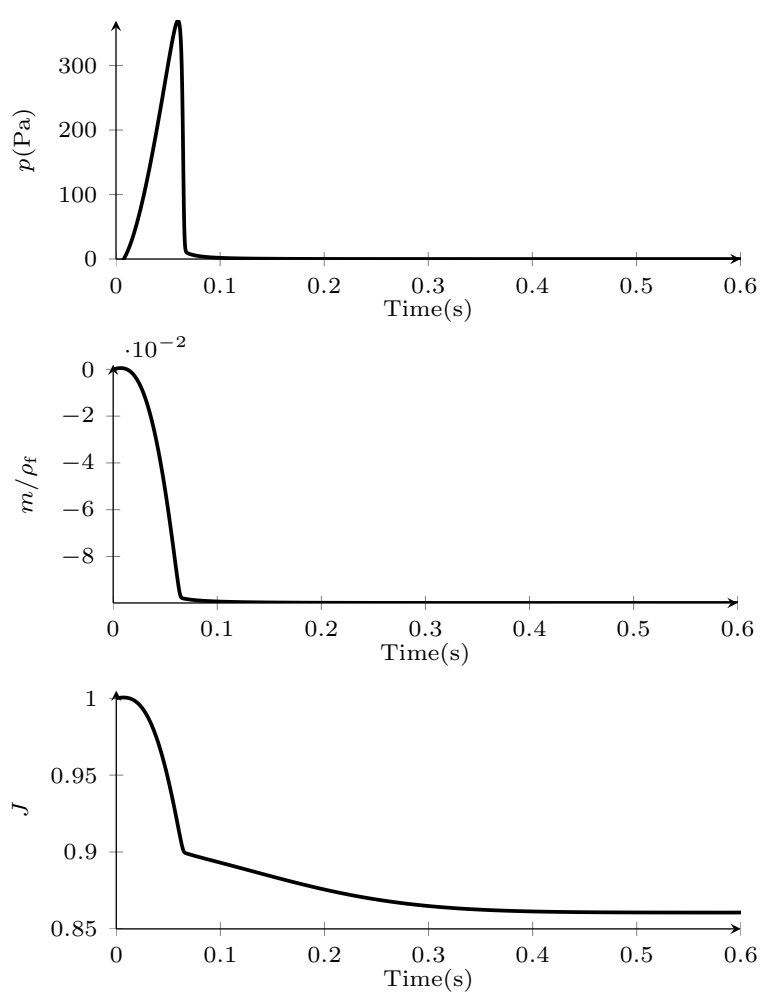

Figure 2: Drainage test. Pressure, fluid mass and jacobian of deformation in time

\section{Remark 9}

In the end of this simulation, $m$ is stable and the denominator of (9b) vanishes. In an in-house finite element software, a test could be performed at quadrature points in order to replace, when it is not well defined, the finite difference definition of $p(9 \mathrm{~b})$ by its asymptotic expression. As FreeFem ++ does not allow this, we used in this simulation for the pressure law the mid-point rule

$$
\frac{p^{n+1}}{\rho_{\mathrm{f}}}=\frac{1}{2} \pi_{m}\left[\frac{\partial \Psi}{\partial m}\left(J^{n}, m^{n+1}\right)+\frac{\partial \Psi}{\partial m}\left(J^{n}, m^{n}\right)\right] .
$$

\subsection{Energy balance monitoring}

We consider here a test case that satisfies the assumptions of the stability analysis, with no external loading, and no energy sources, but only an initial deformation prescribed on the skeleton (of about 20\%). Geometry and boundary conditions are the same as in the drainage test, but the fluid remains in the skeleton as there is no volume-distributed fluid source nor sink. The monitoring of the energy increment gives an illustration of Proposition 2 and of the various perturbations induced in the energy balance by spatial discretization, see Fig. 3. We observe that the departures from exact energy stability - namely, when the energy rate is positive - are very limited, and appear to be primarily explained by the perturbation $\mathcal{S}_{3}$ induced in the GCL due to computing $\phi$ by projection instead of exact expression at quadrature points. Of course, this perturbation could be easily removed in an in-house finite element software. 

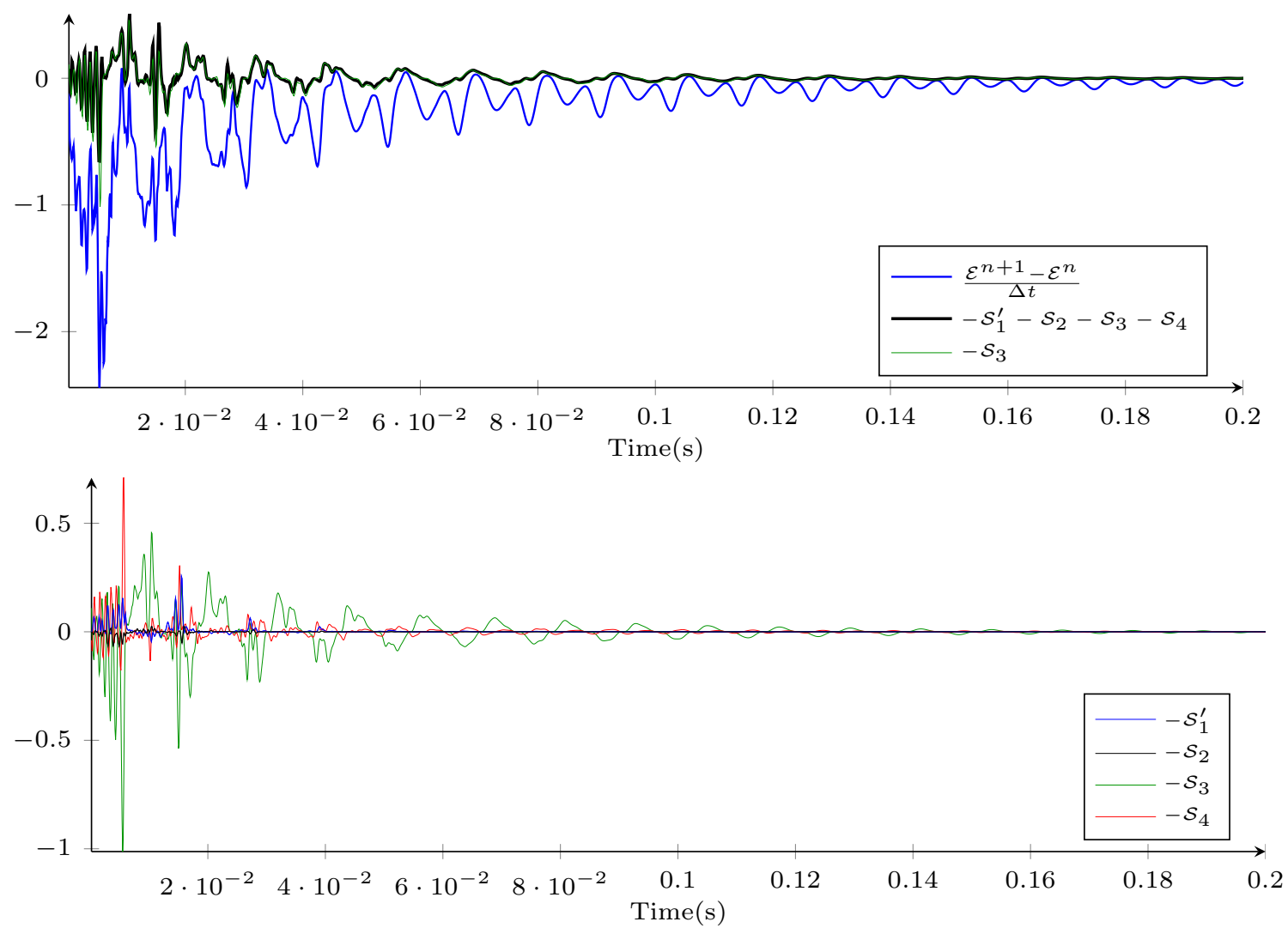

Figure 3: Energy balance monitoring in a homogeneous test case. Top: bound of the energy increment by the consistency term $-\mathcal{S}_{1}^{\prime}-\mathcal{S}_{2}-\mathcal{S}_{3}-\mathcal{S}_{4}$ in time, according to (24), and $-\mathcal{S}_{3}$ alone - Bottom: plot of the separate contributions $-\mathcal{S}_{1}^{\prime},-\mathcal{S}_{2},-\mathcal{S}_{3}$ and $-\mathcal{S}_{4}$; note that $\mathcal{S}_{3}$, and then $\mathcal{S}_{4}$, are an order of magnitude larger than $\mathcal{S}_{1}$ and $\mathcal{S}_{2}$ 


\section{Concluding remarks}

We have proposed an effective partitioned time scheme adapted to the poromechanics formulation of [10], and established a discrete energy estimate for this time scheme. This energy estimate is consistent with the continuous energy balance, up to some numerical dissipation effects, and some perturbations that have been carefully identified and numerically assessed. Among these perturbations, our assessment reveals that the major effect - albeit quite limited quantitatively - lies in a departure from a GCL type property that can be easily treated by evaluating the fluid fraction quantity at the Gauss quadrature points, when this is possible in the finite element software.

In addition, we have provided some numerical illustrations of our numerical strategy by reproducing some test cases proposed in [9], with typical features of large strains and rapid flows as enabled by our general poromechanical formulation, and also a singular transition related to total drainage in the second example.

Further work will focus on spatial discretization issues, and in particular on the treatment of numerical locking - and associated pressure instabilities - phenomena that arise when the solid behavior approaches incompressibility.

Acknowledgement: The authors are grateful to Miguel Angel Fernández (Inria) for some insightful discussions on partitioned methods with Robin coupling conditions.

\section{References}

[1] M. Astorino, F. Chouly, and M.A. Fernández. Robin based semi-implicit coupling in fluidstructure interaction: stability analysis and numerics. SIAM J. Sci. Comput., 31(6):40414065, 2009.

[2] M. Astorino, J.F. Gerbeau, O. Pantz, and K.F. Traoré. Fluid-structure interaction and multi-body contact: Application to aortic valves. Computer Methods in Applied Mechanics and Engineering, 198(45-46):3603-3612, 2009.

[3] S. Badia, F. Nobile, and C. Vergara. Fluid-structure partitioned procedures based on Robin transmission conditions. Journal of Computational Physics, 227(14):7027-7051, 2008.

[4] S. Badia, A. Quaini, and A. Quarteroni. Modular vs. non-modular preconditioners for fluidstructure systems with large added-mass effect. Computer Methods in Applied Mechanics and Engineering, 197(49-50):4216-4232, 2008.

[5] S. Badia, A. Quaini, and A. Quarteroni. Coupling Biot and Navier-Stokes equations for modelling fluid-poroelastic media interaction. Journal Of Computational Physics, 228(21):7986-8014, 2009.

[6] M.A. Biot. Theory of finite deformations of porous solids. Indiana University Mathematics Journal, 21(7):597-620, 1972.

[7] E. Burman and M.A Fernández. Stabilization of explicit coupling in fluid-structure interaction involving fluid incompressibility. Computer Methods In Applied Mechanics And Engineering, 2009.

[8] P. Causin, J.F. Gerbeau, and F. Nobile. Added-mass effect in the design of partitioned algorithms for fluid-structure problems. Comput Method Appl M, 194(42-44):4506-4527, 2005. 
[9] D. Chapelle, J.F. Gerbeau, J. Sainte-Marie, and I.E. Vignon-Clementel. A poroelastic model valid in large strains with applications to perfusion in cardiac modeling. Computational Mechanics, 46(1):91-101, 2009.

[10] D. Chapelle and P. Moireau. General coupling of porous flows and hyperelastic formulations-From thermodynamics principles to energy balance and compatible time schemes. European Journal of Mechanics - B/Fluids, 46:82-96, 2014.

[11] A.J. Chorin. A numerical method for solving incompressible viscous flow problems. $J$. Comput. Phys., 2(1):12-26, 1967.

[12] P.G. Ciarlet. Mathematical Elasticity, Volume I: Three-Dimensional Elasticity. NorthHolland, 1988.

[13] O. Coussy. Poromechanics. John Wiley and Sons, 2004.

[14] R. de Boer. Trends in Continuum Mechanics of Porous Media. Springer, 2005.

[15] J. Donea, S. Giuliani, and J.P. Halleux. An arbitrary Lagrangian-Eulerian finite element method for transient dynamic fluid-structure interactions. Comp. Meth. Appl. Mech. Eng., pages $689-723,1982$.

[16] C. Farhat, P. Geuzaine, and C. Grandmont. The Discrete Geometric Conservation Law and the Nonlinear Stability of ALE Schemes for the Solution of Flow Problems on Moving Grids. J Comput Phys, 174(2):669-694, 2001.

[17] C. Farhat, M. Lesoinne, and P. Le Tallec. Load and motion transfer algorithms for fluid/structure interaction problems with non-matching discrete interfaces: Momentum and energy conservation, optimal discretization and application to aeroelasticity. Computer Methods in Applied Mechanics and Engineering, 157(1-2):95-114, 1998.

[18] C.A. Felippa, K.C. Park, and C. Farhat. Partitioned analysis of coupled mechanical systems. Computer Methods in Applied Mechanics and Engineering, 190(24-25):3247-3270, 2001.

[19] M.A. Fernández, J.F. Gerbeau, and C. Grandmont. A projection semi-implicit scheme for the coupling of an elastic structure with an incompressible fluid. International Journal for Numerical Methods in Engineering, 69(4):794-821, January 2007.

[20] L. Formaggia, A. Quarteroni, and A. Veneziani. Cardiovascular Mathematics: Modeling and Simulation of the Circulatory System, volume 1. Springer Science \& Business Media, 2010 .

[21] J.F. Gerbeau and M. Vidrascu. A Quasi-Newton Algorithm Based on a Reduced Model for Fluid-Structure Interaction Problems in Blood Flows. ESAIM: M2AN, 37(4):631-647, 2003.

[22] O. Gonzalez. Exact energy and momentum conserving algorithms for general models in nonlinear elasticity. Computer Methods in Applied Mechanics and Engineering, 190(1314):1763-1783, 2000.

[23] J.L. Guermond, P. Minev, and J. Shen. An overview of projection methods for incompressible flows. Comput Method Appl M, 195(44-47):6011-6045, September 2006. 
[24] H. Guillard and C. Farhat. On the significance of the geometric conservation law for flow computations on moving meshes. Computer Methods in Applied Mechanics and Engineering, 190(11):1467-1482, 2000.

[25] P. Hauret and P. Le Tallec. Energy-controlling time integration methods for nonlinear elastodynamics and low-velocity impact. Comput Method Appl M, 195(37-40):4890-4916, 2006 .

[26] F. Hecht. New development in FreeFem++. J. Numer. Math., 20(3-4):251-265, 2012.

[27] M. Heil, A.L. Hazel, and J. Boyle. Solvers for large-displacement fluid-structure interaction problems: Segregated versus monolithic approaches. Computational Mechanics, 43(1):91$101,2008$.

[28] U. Küttler, M. Gee, C. Förster, A. Comerford, and W.A. Wall. Coupling strategies for biomedical fluid-structure interaction problems. International Journal for Numerical Methods in Biomedical Engineering, 26(3-4):305-321, 2010.

[29] U. Küttler and W.A. Wall. Fixed-point fluid-structure interaction solvers with dynamic relaxation. Computational Mechanics, 43(1):61-72, 2008.

[30] L. Lanoye, J. Vierendeels, P. Segers, and P. Verdonck. Vascular fluid-structure-interaction using fluent and abaqus software. Journal of Biomechanics, 39:S440, 2006.

[31] P. Le Tallec. Numerical methods for nonlinear three-dimensional elasticity. In P.G. Ciarlet and J.L. Lions, editors, Handbook of Numerical Analysis, Vol. III. Elsevier, 1994.

[32] P. Le Tallec and P. Hauret. Energy conservation in fluid structure interactions. In O. Pironneau Y. Kuznetsov, P. Neittanmaki, editor, Numerical Methods for Scientific Computing / Variational Problems and Applications - CIMNE Barcelona, 2003.

[33] P. Le Tallec and J. Mouro. Fluid structure interaction with large structural displacements. Computer Methods in Applied Mechanics and Engineering, 190(24-25):3039-3067, 2001.

[34] B. Markert, Y. Heider, and W. Ehlers. Comparison of monolithic and splitting solution schemes for dynamic porous media problems. Int. J. Numer. Meth. Engng., 82(11):1341$1383,2010$.

[35] H.G. Matthies and J. Steindorf. Partitioned but strongly coupled iteration schemes for nonlinear fluid-structure interaction. Computers and Structures, 80:1991-1999, 2002.

[36] J. Nitsche. Über ein Variationsprinzip zur Lösung von Dirichlet-Problemen bei Verwendung von Teilräumen, die keinen Randbedingungen unterworfen sind. Abh. Math. Sem. Univ. Hamburg, 36:9-15, 1971.

[37] F. Nobile. Numerical approximation of fluid-structure interaction problems with application to haemodynamics. PhD thesis, EPFL, 2001.

[38] F. Nobile and L. Formaggia. A Stability Analysis for the Arbitrary Lagrangian-Eulerian Formulation with Finite Elements. East-West Journal of Numerical Mathematics, 7(2):105$131,1999$.

[39] R. Temam. Une méthode d'approximation de la solution des équations de Navier-Stokes. Bull. Soc. Math., France 96:115-152, 1968. 
[40] P.D. Thomas and C.K. Lombard. Geometric conservation law and its application to flow computations on moving grids. AIAA Journal, 17(10):1030-1037, 1979.

[41] A.T. Vuong, L. Yoshihara, and W.A. Wall. A general approach for modeling interacting flow through porous media under finite deformations. Computer Methods In Applied Mechanics And Engineering, 283:1240-1259, 2015. 